\title{
Weak lensing with COMBO-17: estimation and removal of intrinsic alignments
}

\author{
Catherine Heymans $^{1 \star}$, Michael Brown ${ }^{2}$, Alan Heavens ${ }^{2}$, Klaus Meisenheimer ${ }^{3}$, \\ Andy Taylor ${ }^{2} \&$ Christian Wolf ${ }^{1}$ \\ ${ }^{1}$ University of Oxford, Astrophysics, Keble Road, Oxford, OX1 3RH, UK \\ ${ }^{2}$ Institute for Astronomy, University of Edinburgh, Royal Observatory, Blackford Hill, Edinburgh, EH9 $3 H J$, UK \\ ${ }^{3}$ Max-Planck-Institut für Astronomie, Königstuhl, D-69117, Heidelberg, Germany
}

6 November 2018

\begin{abstract}
We estimate and remove the contamination of weak gravitational lensing measurements by the intrinsic alignment of close pairs of galaxies. We do this by investigating both the aperture mass B mode statistic, and the shear correlations of close and distant pairs of galaxies. These can be used to quantify non-lensing effects in weak lensing surveys. We re-analyse the COMBO-17 survey, and study published results from the Red-sequence Cluster Survey and the VIRMOS-DESCART survey, concluding that the intrinsic alignment effect is at the lower end of the range of theoretical predictions. We also revisit this theoretical issue, and show that misalignment of baryon and halo angular momenta may be an important effect which can reduce the intrinsic ellipticity correlations estimated from numerical simulations to the level that we and the SuperCOSMOS survey observe. We re-examine the cosmological parameter estimation from the COMBO-17 survey, using the shear correlation function, and now marginalising over the Hubble constant. Assuming no evolution in galaxy clustering, and marginalising over the intrinsic alignment signal, we find the mass clustering amplitude is reduced by 0.03 to $\sigma_{8}\left(\Omega_{m} / 0.27\right)^{0.6}=0.71 \pm 0.11$, where $\Omega_{m}$ is the matter density parameter. We consider the forthcoming SuperNova/Acceleration Probe wide weak lensing survey (SNAP), and the Canada-France-Hawaii Telescope Legacy wide synoptic survey, and expect them to be contaminated on scales $>1$ arcmin by intrinsic alignments at the level of $\sim 1 \%$ and $\sim 2 \%$ respectively. Division of the SNAP survey for lensing tomography significantly increases the contamination in the lowest redshift bin to $\sim 7 \%$ and possibly higher. Removal of the intrinsic alignment effect by the downweighting of nearby galaxy pairs will therefore be vital for SNAP.
\end{abstract}

Key words: cosmology: observations - gravitational lensing - large scale structure, galaxies: formation

\section{INTRODUCTION}

The detection of weak gravitational lensing by large scale structure is a direct way to measure the total matter distribution in the Universe, demanding no assumptions for how luminous matter traces the dominant, largely unknown, dark matter component. Detected by several groups, weak gravitational lensing is now a well established technique, used successfully to set joint constraints on the matter density parameter $\Omega_{m}$ and the amplitude of the matter power spectrum, $\sigma_{8}$, (R.Maoli et al. 2001; Rhodes, Refregier \& Groth 2001; Van Waerbeke et al. 2001; Hoekstra, Yee \& Gladders 2002; Bacon et al. 2003; Jarvis et al. 2003; Brown et al. 2003; Hamana et al. 2002), to measure the bias parameter $b$ (Hoekstra, Yee \& Gladders 2001; Pen et al. 2003), and has recently been used

^ heymans@mpia.de to directly extract the 3D non-linear matter power spectrum $P_{\delta}(k)$ (Tegmark \& Zaldarriaga 2002; Pen et al. 2003). Combined with cosmic microwave background observations, weak lensing can provide strong constraints for $\sigma_{8}$ and $\Omega_{m}$ as the degeneracies in each measurement are almost orthogonal in the $\sigma_{8}-\Omega_{m}$ plane (Brown et al. 2003; Contaldi, Hoekstra \& Lewis 2003).

Unlike many other tests of cosmology, weak lensing surveys with photometric redshift information can tightly constrain cosmological quintessence models and the equation of state parameter $w$, which will be key to our understanding of dark energy, (Heavens 2003; Refregier et al. 2003; Benabed \& Van Waerbeke 2003; Jain \& Taylor 2003). With the increased image resolution available from multi-colour space-based lensing surveys it will also be possible to construct high-resolution projected dark matter maps, and 3D dark matter maps of mass concentrations $>1 \times 10^{13} M_{\odot}$ (Taylor 2001; Hu \& Keeton 2002; Bacon \& Taylor 2003; Massey 
et al. 2003). With future deeper and wider multi-colour surveys, for example the Canada-France-Hawaii Telescope Legacy Survey (CFHTLS) (www.cfht.hawaii.edu/Science/CFHTLS) and the spacebased SuperNova/Acceleration Probe (SNAP) (snap.lbl.gov), weak gravitational lensing will soon reach its 'era of high precision cosmology', provided it can get a good handle on the many causes of systematic errors that arise when trying to detect this minute weak lensing signal.

Sources of systematic errors in weak lensing analysis arise from the shearing and smearing of galaxy images caused by the atmosphere, telescope optics, and detectors (Kaiser, Squires \& Broadhurst 1995; hereafter KSB; Luppino \& Kaiser 1997). With excellent seeing observing conditions, or space-based data, combined with instruments and detectors that are designed with weak lensing detection in mind, these effects can be minimised and corrected for (see for example Rhodes et al. 2003 and Bacon, Refregier $\&$ Ellis 2000). Aside from these observational effects there is a potentially significant error arising from a key assumption made for all weak lensing studies, that galaxy ellipticities are randomly oriented on the sky. Gravitational interactions during galaxy formation could however produce intrinsic shape correlations between nearby galaxies, mimicking to an extent weak lensing shear correlations. As the new generation of wide-field deep weak lensing surveys beat down their observational sources of systematics, it is this additional source of intrinsic ellipticity correlations that could limit the accuracy of cosmological parameter estimation from weak lensing studies. The extent to which this is true will be aided by the study in this paper.

Observational evidence for the existence of intrinsic galaxy alignments comes from the detection of galaxy ellipticity correlations in low redshift surveys where weak lensing shear correlations are negligible, for example in the SuperCOSMOS survey (Brown et al. 2002), and in the Tully catalogue (Lee, Pen \& Seljak 2002). Theoretically, the intrinsic alignment of nearby galaxies has been investigated through numerical simulations and analytical techniques. These have provided estimates of the order $10 \%$ contamination to weak lensing measurements from surveys with median redshift $z_{m} \sim 1.0$ (Heavens, Refregier \& Heymans 2000, hereafter HRH, Croft \& Metzler 2000; Catelan, Kamionkowski \& Blandford 2001; Crittenden et al. 2001, hereafter CNPT; Lee \& Pen 2001; Hui \& Zhang 2002; Porciani, Dekel \& Hoffman 2002; Jing 2002, hereafter Jing; Mackey, White \& Kamionkowski 2002). Whilst there is broad agreement between these studies on the effect for weak lensing measurements, the finer details can differ by up to an order of magnitude or more, with the numerical simulations generally predicting a higher level of contamination than the semianalytic studies. In this paper, we re-examine this issue, and show that a combination of misalignment of the baryon and halo angular momentum, as determined by van den Bosch et al. (2002), and the finite thickness of disk galaxies, modifies the predictions of HRH bringing them into good agreement with the semi-analytic model of CNPT.

With redshift information, it has been shown that the intrinsic signal can be suppressed in weak lensing analysis by downweighting galaxy pairs which are physically close (Heymans \& Heavens 2003; King \& Schneider 2002). This can be done optimally without significantly increasing the shot noise in the final weak lensing analysis, and for this it is helpful (although not necessary) to have a good estimate of the level of contamination. Obtaining an observationally constrained estimate is therefore one of the purposes of this paper, using three weak lensing surveys: a re-analysis of COMBO-17 (Brown et al. 2003), and the published results of the
Red-sequence Cluster Survey (RCS; Hoekstra et al. 2002) and the VIRMOS-DESCART survey (Van Waerbeke et al. 2002).

This paper is organised as follows. In Section 2 we review three different theoretical models for the intrinsic ellipticity correlations between nearby galaxies, taken from HRH, Jing and CNPT. We present a modification to the HRH analysis that we apply to numerical simulations in Section 2.2, finding excellent agreement with the observed intrinsic alignment signal from the SuperCOSMOS survey. Using the three different intrinsic alignment models: HRH, Jing, CNPT, and our modified HRH model which we shall call $\mathrm{HRH}^{*}$ hereafter, we then determine intrinsic alignment contributions to the aperture mass B mode statistic $M_{\perp}$ (Schneider et al. 1998), for the RCS and the VIRMOS-DESCART survey. Using published measurements of $M_{\perp}$ as upper limits for the intrinsic galaxy alignment contribution, we show in Section 3 that, assuming there is no evolution in galaxy clustering, we can reject the intrinsic alignment models of Jing and HRH. In Section 4, we observationally constrain and remove the contribution to COMBO-17's weak lensing measurements by intrinsic galaxy alignments. This is made possible due to the highly accurate photometric information of the COMBO-17 survey, through the application of an optimal intrinsic alignment contamination removal method as described in Heymans \& Heavens (2003). In Section 5, we investigate the effect that galaxy clustering evolution would have on our results. We look at the implications for weak lensing analysis in Section 6, constraining $\sigma_{8}$ and $\Omega_{m}$ with the shear correlation function statistic from COMBO-17, where we now include marginalisation over our measured intrinsic alignment signal. We also determine estimates of the contamination of shear correlation measurements from the CFHTLS and SNAP surveys, summarising and concluding in Section 7.

\section{INTRINSIC ELLIPTICITY CORRELATIONS}

The ellipticity of a galaxy approximated as an ellipse with axial ratio $q$, at position angle $\phi$ measured counter-clockwise from the $x$ axis, can be defined as

$\left(\begin{array}{l}e_{1} \\ e_{2}\end{array}\right)=\frac{q^{2}-1}{q^{2}+1}\left(\begin{array}{c}\cos 2 \phi \\ \sin 2 \phi\end{array}\right)$.

In the weak lensing régime the observed (complex) ellipticity $e$ is related to the source ellipticity $e^{s}$ and the complex shear $\gamma$ by $e \simeq$ $e^{s}+2 \gamma$. With many source galaxies the shear can be estimated from

$\gamma \simeq \frac{1}{2}\langle e\rangle \equiv \frac{1}{2}\left\langle e_{1}+i e_{2}\right\rangle$.

See Rhodes, Refregier \& Groth (2000) for an exact relationship. A measurement of the shear correlation function $\left\langle\gamma \gamma^{*}\right\rangle$ can be directly related to the matter power spectrum (see Bartelmann \& Schneider 2001 and references therein), but to estimate it observationally includes an uncertain contribution from intrinsic correlations between the ellipticities of nearby galaxy pairs $I_{a b} \equiv e_{a}^{s} e_{b}^{s *}$ if, when averaging over many galaxy pairs, $\left\langle I_{a b}\right\rangle \neq 0$. Hence the expectation of the shear correlation function is given by

$E\left[\gamma \gamma^{*}: \theta\right]=\frac{1}{4} \sum_{a b}\left(e_{a}(\mathbf{x}) e_{b}^{*}(\mathbf{x}+\theta)-I_{a b}\right)$.

Several groups have found theoretical support for non-zero intrinsic correlations, and the existing methods used to determine its amplitude can be broadly split into two approaches. The first employs numerical simulations with varying simplifying assumptions, relating galaxy shape to dark matter halo properties. The second uses 
analytic techniques, relating ellipticity correlations to initial linear tidal shear field correlations. These methods approximate $I_{a b}$ as a theoretically determined three-dimensional ellipticity correlation function $\eta(r)$ which depends only on the comoving distance between the galaxy pair $r \equiv|\mathbf{r}|$ :

$\eta(r)=\left\langle e(\mathbf{x}) e^{*}(\mathbf{x}+\mathbf{r})\right\rangle$.

We project $\eta(r)$ into two dimensions using a modified Limber equation, in order to compare it with observed angular ellipticity correlations. In the absence of weak gravitational lensing, for example with the low redshift SuperCOSMOS data (Brown et al. 2002), measured angular ellipticity correlations can be directly compared to those predicted from theoretical models:

$C_{I}(\theta)=\frac{\int d z_{a} d z_{b} \phi_{z}\left(z_{a}\right) \phi_{z}\left(z_{b}\right)\left[1+\xi_{g g}\left(r_{a b}\right)\right] \eta\left(r_{a b}\right)}{\int d z_{a} d z_{b} \phi_{z}\left(z_{a}\right) \phi_{z}\left(z_{b}\right)\left[1+\xi_{g g}\left(r_{a b}\right)\right]}$,

where $\phi_{z}\left(z_{a}\right)$ is either a known redshift distribution, for example the COMBO-17 photometric redshift distribution shown in Fig. A1 of the Appendix, or can be approximated by

$\phi_{z}(z) \propto z^{2} \exp \left[-\left(\frac{z}{z_{0}}\right)^{1.5}\right]$,

for a survey with median redshift $z_{m} \approx 1.4 z_{0}$. Most studies have found $\eta\left(r_{a b}\right)$ to be significantly non-zero only for galaxies closer than a few tens of Mpc, where the comoving separation, $r_{a b}$ is given accurately enough in a flat universe by

$r_{a b}^{2} \simeq\left(w_{a}-w_{b}\right)^{2}+\left(\frac{w_{a}+w_{b}}{2}\right)^{2} \theta^{2}$,

where $w$ is the comoving radial geodesic distance. The signal depends on the galaxy two-point correlation function, $\xi_{g g}(r)$, since it determines how many galaxy pairs observed at a given angular separation are physically close together. We take the correlation function to be non-evolving (Cole et al. 1994; Giavalisco et al. 1998; Adelberger et al. 1998):

$\xi_{g g}(r)=\left(\frac{r}{r_{0}}\right)^{\gamma}$

with $\gamma=-1.8$ and $r_{0}=5 h^{-1} \mathrm{Mpc}$. In Section 5 we will also consider the effect of redshift dependence in the galaxy correlation function, as claimed by Le Fevre et al. (1996); Postman et al. (1998); Baugh et al. (1999); Carlberg et al. (2000); Firth et al. (2002); Wilson (2003).

In this Section we will focus on three studies: HRH and Jing, which are based on numerical simulations, and CNPT, where the strength of ellipticity correlations are analytically computed in linear theory. Fig. 1 compares three published functional fits, defined in Section 2.1, for the three-dimensional ellipticity correlation functions, $\eta(r)$, showing that whilst there is some rough agreement in the results from these studies, the numerical simulations in general, predict a stronger intrinsic alignment effect compared to the analytical technique.

\subsection{Intrinsic Alignment Models}

Assuming that luminous matter forms galaxies in all dark matter halos above a minimum mass limit, $M_{h}$, it is possible to acquire a three-dimensional catalogue of galaxy shapes from a large N-body dark matter simulation. HRH have modelled the shape of each luminous galaxy as an infinitesimally thin disc placed perpendicular to its angular momentum vector, which is assumed to be perfectly

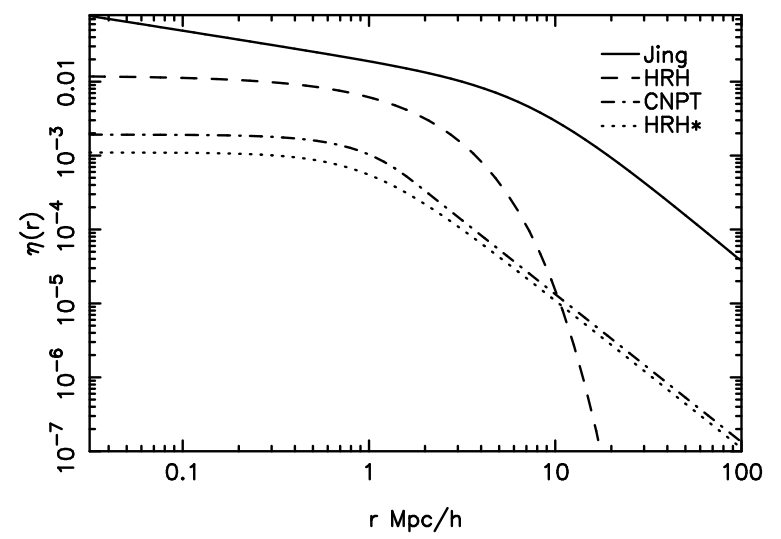

Figure 1. Intrinsic ellipticity correlation models. Comparison of different functional fits to three intrinsic alignment studies, Jing (solid), HRH (dashed) and CNPT (dot dash). The modified HRH model that is measured and defined in Section 2.2 is also plotted (dotted).

aligned with that of its parent dark matter halo. This resulted in ellipticity correlations that were fitted with an exponential

$\eta_{\mathrm{HRH}}(r)=0.012 \exp \left(-\frac{r}{1.5 h^{-1} M p c}\right)$.

Using a higher resolution N-body simulation, Jing assumed that the galaxy ellipticity was equal to that of its parent dark matter halo, resulting in ellipticity correlations with a best fitting functional form defined for $\left\langle e_{1} e_{1}\right\rangle$,

$\eta_{\text {Jing }}(r) \approx 2\left\langle e_{1} e_{1}\right\rangle=2 \frac{3.6 \times 10^{-2}\left(\frac{M_{h}}{10^{10} h^{-1} M p c}\right)^{0.5}}{r^{0.4}\left(7.5^{1.7}+r^{1.7}\right)}$,

where in this paper we will use $M_{h}=6.9 \times 10^{11} M_{\odot}$, which corresponds to the minimum mass limit used by HRH. HRH found a similar shape for the ellipticity correlations of dark matter haloes. CNPT related galaxy ellipticity correlations to the initial correlations in the tidal shear field. Assuming that the density field has a Gaussian distribution, CNPT found that the ellipticity correlation is well approximated at large $r \gtrsim 3 h^{-1} \mathrm{Mpc}$ by

$\eta_{\mathrm{CNPT}}(r)=\frac{a^{2} \beta^{2}}{84} \frac{\varepsilon^{2}(r)}{\varepsilon^{2}(0)}$

where $a$ quantifies the uncertainty in the correlation between the shear field and a galaxy's moment of inertia, $\beta$ accounts for the nonzero thickness of galaxy disks where $e_{\text {gal }}=\beta e_{\text {thin disc }}$, and $\varepsilon(r)$ is a density correlation function. For the purpose of this paper, we use an analytic solution to equation (11) for the simple model proposed in CNPT, who took $a=0.55$, inferred from numerical simulation results (Heavens, Refregier \& Heymans 2000), $\beta=0.73$, from the mean of the measured ellipticity distribution of the APM survey (Lambas, Maddox \& Loveday 1992) and from the observed distribution of ellipticities measured for lensing studies (Ebbels 1998), and $\varepsilon(r) \propto 1 / r$, where we also follow CNPT by including top-hat smoothing on $1 h^{-1} \mathrm{Mpc}$ scales.

\subsection{Intrinsic alignments of galaxies: revisiting theoretical expectations}

Recent results from numerical simulations of structure formation by van den Bosch et al. (2002), have shown that the angular momentum of non-radiative gas and dark matter are actually poorly 


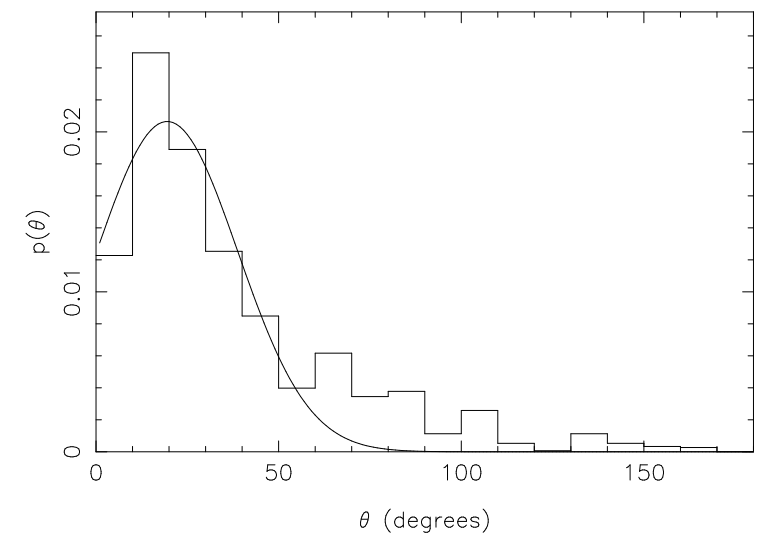

Figure 2. Distribution of the misalignment angle between the total angular momentum vectors of the dark matter and gas, as found by van den Bosch et al. (2002). Over-plotted is the best fit Gaussian model with $\mu=19.5^{\circ}$ and $\sigma=19.3$.

aligned with a median misalignment angle of $\sim 30^{\circ}$. Observational support for some misalignment, albeit smaller, comes from strong lensing considerations in the CASTLES survey (Keeton, Kochanek $\&$ Falco 1998). With these results we are able to advance the analysis of HRH, where instead of assuming perfect alignment between the angular momentum of the halo and baryons, we include a probability distribution for the misalignment. Figure 2 shows the probability distribution of the polar misalignment angle $\theta$ as found by van den Bosch et al. (2002) and the best fit Gaussian model. van den Bosch et al. (2002) note that higher resolution simulations are required to reduce the possible impact of discreteness effects on this probability distribution, but they do conclude that there is a true misalignment that is significantly different from zero.

In light of these results we have reanalysed the N-body simulation developed by the Virgo Consortium (Jenkins et al. 1998), for a $\Lambda$ CDM cosmological model, $\left(\Omega_{m}=0.3, \Omega_{\Lambda}=0.7, \sigma_{8}=0.9\right.$, $\Gamma=0.2$ ), approximating the probability distribution for the misalignment polar angle as found by van den Bosch et al. (2002), as a Gaussian with mean $\mu=20^{\circ}$ and width $\sigma=20^{\circ}$, which is truncated at zero misalignment. We assign a random misalignment azimuthal angle around the original halo angular momentum vector. The galaxy ellipticity is then determined from the angular momentum of the baryons, by,

$\left|e\left(L_{z}\right)\right|=\beta\left(\frac{1-L_{z}^{2}}{1+L_{z}^{2}}\right)$,

where we now account for galaxy thickness, following CNPT by taking $\beta=0.73$. Fig. 3 compares the resulting 3D ellipticity correlation function $\eta(r)$, with the HRH model from equation (9), (dashed), showing that with the inclusion of baryon halo angular momentum misalignment, the amplitude of the galaxy alignments predicted from numerical simulations is significantly reduced. We choose to fit a simple function

$\eta(r)=\frac{A}{1+(r / B)^{2}}$.

We fix $B=1 h^{-1} \mathrm{Mpc}$, which is similar to the CNPT choice of a smoothing length of $1 h^{-1} \mathrm{Mpc}$, and calculate the maximum likelihood value for the amplitude $A$. A small positive value is preferred, although as shown dot-dashed in Fig. 10, it should be noted that the reduced signal from the misalignment means the data are actually consistent with zero. The best fit shown dashed in Fig. 3 is ( $r$ in $\left.h^{-1} \mathrm{Mpc}\right)$

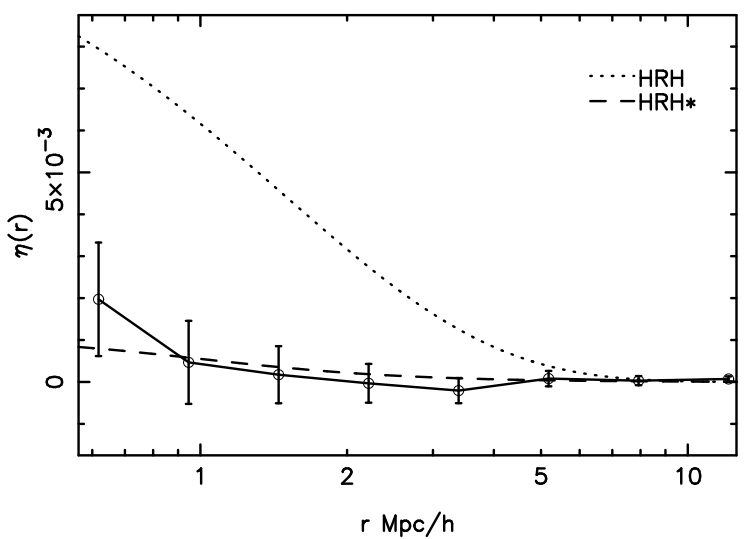

Figure 3. $3 \mathrm{D}$ ellipticity correlation function estimated from the $\Lambda \mathrm{CDM}$ Virgo simulation, assuming that the gas disc and dark matter halo are misaligned. Over-plotted is the HRH model $\eta_{\mathrm{HRH}}(r)$ (dotted) and the best fit HRH modified model $\eta_{\mathrm{HRH} *}(r)$ (dashed).

$\eta_{\mathrm{HRH} *}(r)=\frac{0.0011}{1+r^{2}}$,

which is consistent with the CNPT analytical model. Note that the new model fit lies above the old HRH model at large scales. This should not be taken to imply that misalignment increases the correlations, rather, it reflects the fact that the old exponential fit of HRH underestimated the (noisy) correlations on large scales.

Comparing our $\mathrm{HRH}^{*}$ model with the ellipticity variance measured in the low redshift SuperCOSMOS survey (Brown et al. 2002), we find excellent agreement, especially on scales $>35$ arcmin, see Fig. 4. We have calculated the HRH* angular ellipticity correlation function from equation (5) with a median redshift $z_{m}=0.1$, and related it to the ellipticity variance statistic following Bacon et al. (2003):

$\sigma_{e}^{2}(\theta)_{\square}=\frac{2 \sqrt{\pi}}{\theta^{2}} \int_{0}^{\theta} d \theta^{\prime} \theta^{\prime} C_{I}\left(\theta^{\prime}\right)$,

where the factor $\sqrt{\pi}$ is a good approximation to use in order to scale the ellipticity variance measured in circular apertures of radius $\theta$ to the ellipticity variance as measured in SuperCOSMOS, in square cells of length $\theta$ (Bacon, Refregier \& Ellis 2000). We calculate the likelihood of the HRH* model from the SuperCOSMOS results on scales $\theta>20$ arcmin, allowing the amplitude to vary, see Fig. 10. Below this angular scale the SuperCOSMOS results are extremely noisy and could suffer contamination from residual point spread function anisotropy distortions, (see Brown et al. (2002) for details). We therefore omit these points from the likelihood fitting procedure, finding the best fit amplitude $A=0.0009 \pm 0.0005$, ( $95 \%$ confidence limits).

\section{UPPER LIMITS ON THE INTRINSIC ALIGNMENT SIGNAL: APERTURE MASS B MODES}

A good diagnostic for determining the level of systematic errors present in weak lensing measurements, is to decompose the shear correlation signal into E and B modes. This was first proposed by Crittenden et al. (2002), and is now a standard statistical test for the presence of non-lensing contributions to weak lensing measurements. Weak gravitational lensing produces curl-free distortions (E-type), and contributes only to the B-type distortions at small angular scales, $\theta<1$ arcmin, due to source redshift clustering 


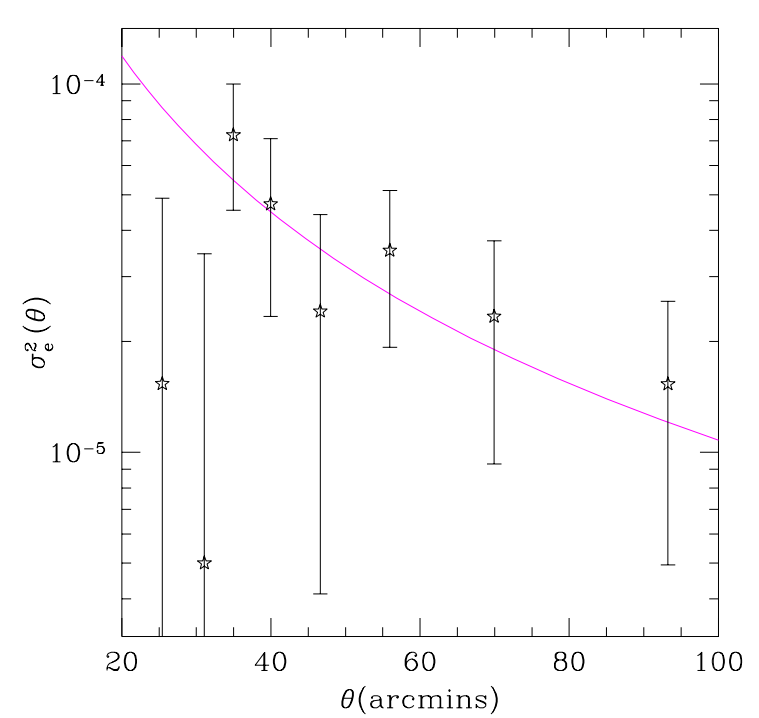

Figure 4. Comparison of the measured ellipticity variance in the SuperCOSMOS survey, see Brown et al. (2002) for details, compared to the ellipticity variance expected at this redshift from the modified HRH model, HRH*.

(Schneider, Van Waerbeke \& Mellier 2002). A significant detection of a B-type signal in weak lensing surveys is therefore an indication that ellipticity correlations exist either from residual systematics within the data and/or from intrinsic galaxy alignments.

The decomposition has previously been carried out by determining the B mode shear power spectrum, $C_{l}^{\beta \beta}$ (Brown et al. 2003; Pen, Van Waerbeke \& Mellier 2002) or by using the aperture mass statistic, $M_{a p}$ (Hoekstra, Yee \& Gladders 2002; Van Waerbeke et al. 2002; Hamana et al. 2002; Jarvis et al. 2003), where the aperture mass statistic is defined to be the aperture weighted dimensionless surface mass density of a lens, $\kappa$ (Kaiser et al. 1994), such that

$M_{\mathrm{ap}}(\theta)=\int_{0}^{\theta} d^{2} \vartheta U(\vartheta) \kappa(\vartheta)$,

where the simplest radial filter function $U(\vartheta)$ is given by,

$U(\vartheta)=\frac{9}{\pi \theta^{2}}\left(1-\frac{\vartheta^{2}}{\theta^{2}}\right)\left(\frac{1}{3}-\frac{\vartheta^{2}}{\theta^{2}}\right)$,

(Schneider et al. 1998). With this choice of filter function the aperture mass $M_{a p}$ is related to the power spectrum by

$\left\langle M_{\mathrm{ap}}^{2}\right\rangle(\theta)=\frac{288}{\pi \theta^{4}} \int_{0}^{\infty} \frac{d k}{k^{3}} P_{\kappa}(k)\left[J_{4}(k \theta)\right]^{2}$,

where $\mathrm{J}_{4}(k \theta)$ is the fourth-order Bessel function of the first kind, and $P_{\kappa}(k)$ is the convergence power spectrum at wave number $k$, related to the non-linear mass power spectrum $P_{\delta}$ by

$P_{\kappa}(k)=\frac{9 H_{0}^{4} \Omega_{m}^{2}}{4 c^{4}} \int_{0}^{w_{H}} d w \frac{g^{2}(w)}{a^{2}(w)} P_{\delta}\left(\frac{k}{f_{K}(w)}, w\right)$,

$a(w)$ is the dimensionless scale factor, $H_{0}$ is the Hubble parameter and $\Omega_{m}$ the matter density parameter. The second argument of $P_{\delta}$ allows for time-evolution of the power spectrum. $g(w)$ is a weighting function locating the lensed sources,

$g(w)=\int_{w}^{w_{H}} d w^{\prime} \phi\left(w^{\prime}\right) \frac{f_{K}\left(w^{\prime}-w\right)}{f_{K}\left(w^{\prime}\right)}$. $\phi(w(z)) d w$ is the observed number of galaxies in $d w$, and $w_{H}$ is the horizon distance (Schneider et al. 1998).

In this paper we will focus on the aperture mass decomposition, which can be directly calculated from angular ellipticity correlation functions as follows:

$\mathrm{E}:\left\langle M_{a p}^{2}\right\rangle=\int_{0}^{\infty} \frac{d \vartheta \vartheta}{2 \theta^{2}}\left[\xi_{+}(\vartheta) T_{+}\left(\frac{\vartheta}{\theta}\right)+\xi_{-}(\vartheta) T_{-}\left(\frac{\vartheta}{\theta}\right)\right]$
$\mathrm{B}:\left\langle M_{\perp}^{2}\right\rangle=\int_{0}^{\infty} \frac{d \vartheta \vartheta}{2 \theta^{2}}\left[\xi_{+}(\vartheta) T_{+}\left(\frac{\vartheta}{\theta}\right)-\xi_{-}(\vartheta) T_{-}\left(\frac{\vartheta}{\theta}\right)\right]$

where,

$\xi_{ \pm}(\vartheta)=\left\langle\gamma_{t} \gamma_{t}\right\rangle_{\vartheta} \pm\left\langle\gamma_{r} \gamma_{r}\right\rangle_{\vartheta} \approx \frac{1}{4}\left(\left\langle e_{t} e_{t}\right\rangle_{\vartheta} \pm\left\langle e_{r} e_{r}\right\rangle_{\vartheta}\right)$

and $T_{ \pm}$are formally given in Crittenden et al. (2002). The tangential and radial ellipticity parameters $e_{t}$ and $e_{r}$ are defined such that:

$\left(\begin{array}{c}e_{t} \\ e_{r}\end{array}\right)=\left(\begin{array}{cc}\cos 2 \phi & \sin 2 \phi \\ -\sin 2 \phi & \cos 2 \phi\end{array}\right)\left(\begin{array}{l}e_{1} \\ e_{2}\end{array}\right)$,

where $\phi$ is defined to be the angle between the $x$ axis and the line joining a galaxy pair.

In the absence of contaminating non-lensing sources we expect $M_{\perp} \approx 0$, but this is found not to be the case in all the weak lensing surveys to date that have measured this diagnostic statistic. We therefore calculate the contribution to the aperture mass B mode from the four intrinsic alignment models defined in Sections 2.1 and 2.2. This is calculated for two different depth weak lensing surveys, the RCS with a median redshift $z_{m} \approx 0.56$, (Hoekstra et al. 2002), and the VIRMOS-DESCART survey with $z_{m} \approx 1.0$, (Van Waerbeke et al. 2002).

Fig. 5 shows the predicted angular shear correlation function, $\left\langle\gamma \gamma^{*}\right\rangle(\vartheta)_{I}=\frac{1}{4} C_{I}(\vartheta)$, for each intrinsic alignment model calculated for both survey depths. We also plot for comparison the shear correlations expected from weak gravitational lensing, for an $\Omega_{m}=0.3, \Omega_{\Lambda}=0.7$ cosmology with a $\Gamma=0.2, \sigma_{8}=0.8$, nonlinear CDM matter power spectrum, $P_{\delta}$ calculated using the fitting formula from Smith et al. (2003):

$\left\langle\gamma \gamma^{*}\right\rangle(\vartheta)_{\mathrm{WGL}}=\frac{1}{2 \pi} \int d k k P_{\kappa}(k) J_{0}(k \vartheta)$.

We calculate the aperture mass B mode contributions from the shear correlation functions shown in Fig. 5, through equation (22). CNPT find that $\left\langle e_{t} e_{t}\right\rangle_{\vartheta} \approx\left\langle e_{r} e_{r}\right\rangle_{\vartheta}$ at small angular scales, deviating slightly at separations $\vartheta>3$ arcmin, and this is consistent with the results we find. We therefore make the simplifying assumption that $\left\langle e_{t} e_{t}\right\rangle_{\vartheta}=\left\langle e_{r} e_{r}\right\rangle_{\vartheta}$ at all angular scales, i.e that there is no preferred intrinsic tangential or radial alignment of galaxy ellipticities, and hence $\xi_{-}=0$ such that

$\left\langle M_{\perp(\mathrm{IA})}^{2}(\theta)\right\rangle=\frac{1}{2} \int_{0}^{\infty} \frac{d \vartheta \vartheta}{\theta^{2}}\left[\xi_{+}(\vartheta) T_{+}\left(\frac{\vartheta}{\theta}\right)\right]$,

where $\xi_{+}(\vartheta)=\frac{1}{4} C_{I}(\vartheta)$ and we use the following analytic expression for $T_{+}(x)$, derived by Schneider, Van Waerbeke \& Mellier (2002), which vanishes at $x>2$ :

$$
\begin{aligned}
T_{+}(x)= & \frac{6\left(12-15 x^{2}\right)}{5}\left[1-\frac{2}{\pi} \arcsin \frac{x}{2}\right]+\frac{x \sqrt{4-x^{2}}}{100 \pi} \\
& \times\left(110+2320 x^{2}-754 x^{4}+132 x^{6}-9 x^{2}\right) .
\end{aligned}
$$

Fig. 6 shows the aperture mass B mode measurements reported by the RCS (Hoekstra, Yee \& Gladders 2002), (upper panel), and VIRMOS-DESCART survey, (Van Waerbeke et al. 2002), (lower 

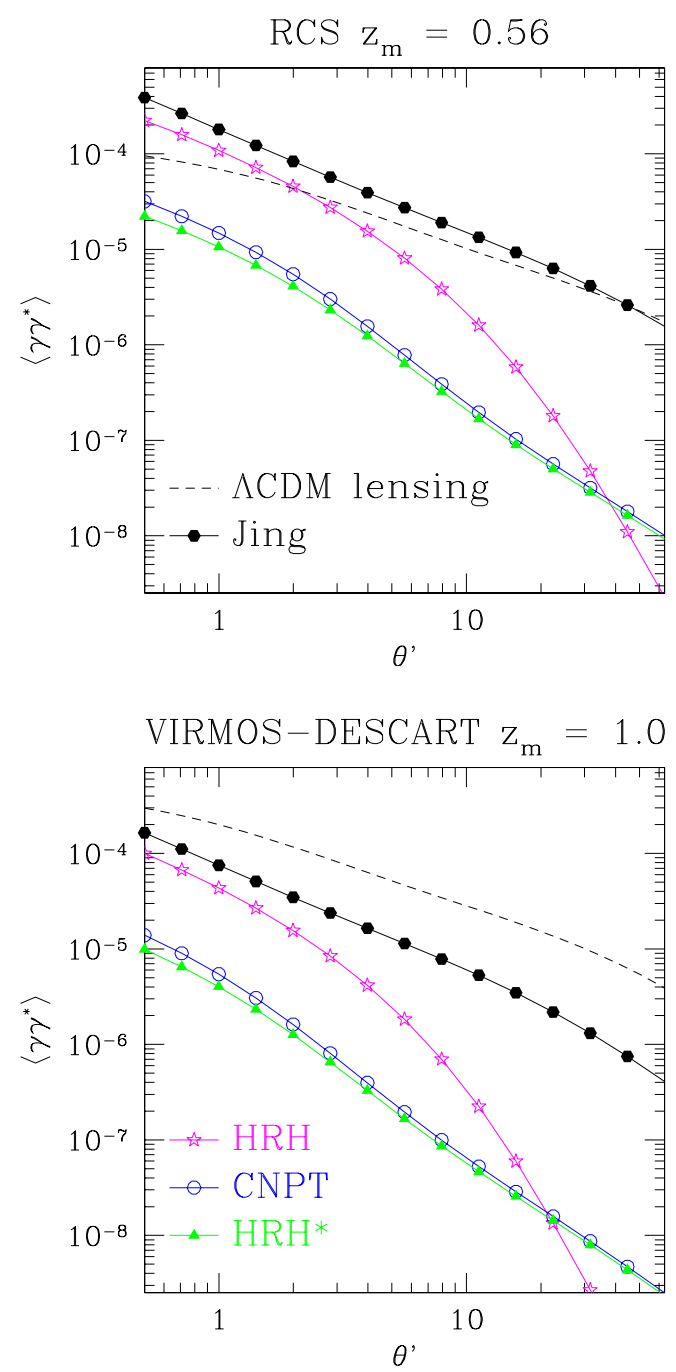

Figure 5. Predicted intrinsic alignment angular shear correlation functions $\left\langle\gamma \gamma^{*}\right\rangle_{I}$, Jing (filled), HRH (stars), CNPT (circles) and HRH* (triangles). The intrinsic alignment contributions are compared to the angular shear correlations from $\Lambda \mathrm{CDM}$ weak gravitational lensing, normalised to $\sigma_{8}=0.8$, (dashed), for the RCS with depth $z_{m}=0.56$, (upper) and for the VIRMOSDESCART survey with depth $z_{m}=1.0$, (lower)

panel), compared to the contributions from the four different intrinsic alignment models. The observed B modes can be attributed in part to residual systematics remaining in the data and in part to intrinsic galaxy alignments. Whilst most models for intrinsic galaxy alignments predict $\mathrm{B}$ modes there are alternatives such as the model proposed by Catelan, Kamionkowski \& Blandford (2001) which does not. Known data systematics introduce positive correlations and we therefore consider these observations as upper limits for intrinsic alignment B modes within these surveys. This is probably a valid assumption on small scales over which the distorting point spread function (PSF) is relatively constant, provided a consistent subtraction method for the PSF has been applied. Highly anisotropic PSF distortions which vary from $e_{i}^{*} \rightarrow-e_{i}^{*}$ across the image could introduce a negative aperture mass B mode on large scales. This could in principle, introduce a systematic negative aperture mass $B$ mode at large scales $\theta>10$ arcmin, prevent-
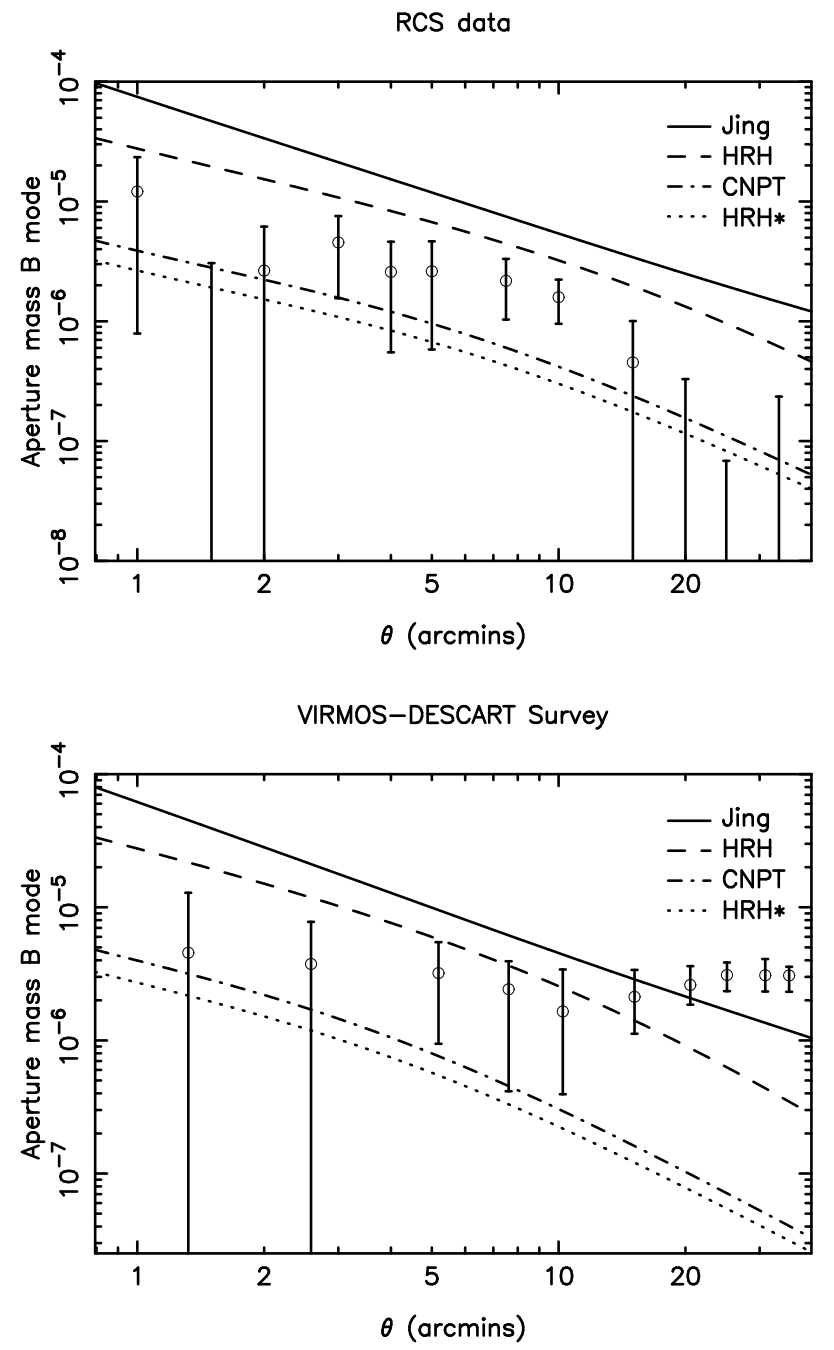

Figure 6. Intrinsic alignment contributions to the aperture mass B mode $\left\langle M_{\perp}^{2}\right\rangle$; Jing(solid), HRH (dashed), CNPT(dot dash) and HRH* (dotted). The upper panel compares intrinsic alignment predictions for the RCS survey with their measured B mode. The lower panel compares intrinsic alignment predictions for the VIRMOS-DESCART with their measured B mode.

ing the use of B modes as an upper limit for intrinsic alignment contamination at these scales.

With no evolution in galaxy clustering, considering the observed B modes as upper limits for $\theta<10$ arcminutes, we find that the Jing and HRH models are strongly rejected by the RCS results and that the CNPT and $\mathrm{HRH}^{*}$ models are favoured by both the VIRMOS-DESCART and the RCS. We note that none of the intrinsic alignment models can account for the significant B mode at large angular scales $\theta>15$ arcmin found in the VIRMOSDESCART data, which may be caused by other data systematics (Hoekstra 2003).

\section{ESTIMATION AND REMOVAL OF THE INTRINSIC ALIGNMENT SIGNAL: SHEAR CORRELATIONS}

Decomposing cosmic shear correlation measurements into $\mathrm{E}$ and $\mathrm{B}$ modes provides an estimate of the intrinsic alignment contamination in weak lensing measurements. However it is not clear how to 
correct the E mode given a measured non-zero B mode. To date the practice has been to add in quadrature the $\mathrm{B}$ mode to the $\mathrm{E}$ mode error (Hoekstra, Yee \& Gladders 2002). An alternative technique, applicable to weak lensing surveys with photometric redshift estimates, is to remove, essentially completely, the intrinsic alignment signal by downweighting pairs of galaxies which are likely to be physically close (King \& Schneider 2002; Heymans \& Heavens 2003, hereafter HH). King \& Schneider (2003) have also proposed separating cosmic shear from intrinsic galaxy alignments using correlation function tomography.

In this Section, we extend the method of $\mathrm{HH}$, to determine the intrinsic alignment signal, as well as to remove it from the shear correlation function. The method is reviewed in the appendix; close galaxy pairs are ignored in the shear correlation analysis, but in the optimised case, no more are removed than is strictly necessary, to avoid needlessly increasing the shot noise.

\subsection{Constraining intrinsic galaxy alignments with COMBO-17: Method}

COMBO-17 is a deep multi-colour wide-field optical survey carried out with the Wide-field Imager (WFI) at the MPG/ESO $2.2 \mathrm{~m}$ telescope that spans 1.25 square degrees to limiting magnitude $R=25.5$ in five separate regions (Wolf et al. 2001). Multi-colour observations through a total of 17 filters, 5 broad-band ( $U B V R I$ ) and 12 narrow band filters ranging from 420 to $914 \mathrm{~nm}$, specifically chosen to facilitate accurate photometric redshift estimation with errors $\Delta_{z} \leq 0.05$ reliable to $R<24$ currently span 1 square degree of the survey area. The WFI instrument consists of a $4 \times 2$ array of $2048 \times 4096$ CCDs with pixel scale $0.238^{\prime \prime}$. Weak lensing studies have been carried out on the deep R-band images observed during the best seeing conditions (Brown et al. 2003), and it is a subset of this R-band selected galaxy sample, limited to $R<24$ with a median redshift $z_{m} \sim 0.6$, that we will use in this analysis. The selected survey area totals 0.75 square degrees in the A901, CDFS and S11 fields, yielding a catalogue of $3.55 \times 10^{4}$ galaxies with KSB shape measurements and photometric redshifts accurate to $\Delta_{z}=0.042$, (see Brown et al. 2003 for further details).

The total observed shear correlation function in the COMBO17 dataset can be expressed as

$C(\theta)=C_{\text {lens }}(\theta)+C_{\mathrm{I}}(\theta)+C_{\text {sys }}(\theta)$,

where $C_{\text {lens }}(\theta)$ is the cosmic shear weak lensing signal $\left\langle\gamma \gamma^{*}\right\rangle$ equation (25), and $C_{\mathrm{I}}(\theta)$ is the signal due to the intrinsic alignment of galaxy shapes equation (5). $C_{\text {sys }}(\theta)$ is the correlation function due to any observational systematic effects which may be present in the dataset (see Brown et al. 2003 for a robust estimate of systematic errors in the data). $C_{\mathrm{I}}(\theta)$ can effectively be eliminated by excluding galaxy pairs which are closer than $\alpha(\theta) \Delta_{z}$ in redshift, where $\Delta_{z}=0.042$ is the typical redshift error for the COMBO17 galaxies and $\alpha(\theta)$ is optimised to minimise the total error from intrinsic alignments and shot noise (see appendix). By measuring the shear correlation function for pairs of galaxies which are sufficiently distant from each other, we exclude intrinsic alignments. Furthermore, if we also measure the shear correlations for only the close galaxy pairs which have been excluded from the analysis, we have two independent correlation functions from which we can estimate the intrinsic alignment signal from the close galaxy pairs:

$C_{\mathrm{IA}}(\theta)=C_{\text {close }}(\theta)-C_{\text {distant }}(\theta)-\Delta C_{\text {lens }}(\theta)$,

where $C_{\text {close }}(\theta)$ is the correlation function measured from the close pairs, $C_{\text {distant }}(\theta)$ is the correlation function for the distant pairs and $\Delta C_{\text {lens }}(\theta)$ is the difference in the correlations caused by weak gravitational lensing for the distant and close pairs. Equation (29) assumes that in the distant data set the intrinsic alignment signal is zero and that $C_{\mathrm{sys}}(\theta)$ is the same as for the close pairs. To ensure all intrinsic alignment contamination has been removed from the distant dataset, we choose the most conservative estimate of $\eta_{\text {Jing }}(r)$ in our $\mathrm{HH}$ weighting scheme. The assumption for $C_{\text {sys }}(\theta)$ is reasonable since there is no reason to believe that systematic effects would depend on the redshift separation of galaxy pairs. To calculate $\Delta C_{\text {lens }}(\theta)$, in Fig. 7 we plot the expected lensing signal for a median redshift $z_{m}=0.6 \Lambda \mathrm{CDM}$ model, normalised to $\sigma_{8}=0.8$, for both the distant-pair dataset and for the close pairs, according to equation (A13) in HH. Although the difference is small, at the $10^{-5}$ level, and could be ignored, we will include it in our analysis assuming that $\Delta C_{\text {lens }}(\theta)$ is approximately the same for each field. The weak lensing signal $C_{\text {lens }}(\theta)$ will however differ significantly between the three fields, A901, CDFS and S11, due to the significant mass concentrations in two of the fields. The S11 field contains a fairly large cluster (Abell 1364) at a redshift of $z=0.11$ while the A901 field includes a supercluster system (Abell 901/902) at $z=0.16$. In the following analysis, we have therefore applied equation (29) to the three fields individually and combined the three resulting measurements of $C_{\mathrm{IA}}(\theta)$ with minimum variance weighting, where the weight $w_{i}$ for each measurement with associated error $\sigma_{i}$ is given by $w_{i}=1 / \sigma_{i}^{2}$. This ensures that $C_{\text {lens }}(\theta)$ is eliminated in the subtraction. Note also that, for the various $C(\theta)$ calculations, we have removed cluster member galaxies from the A901 and S11 fields to eliminate contamination of the field intrinsic alignment signal by intra-cluster galaxy alignments (Plionis et al. 2003).

To estimate $C_{\text {close }}(\theta)$ and $C_{\text {distant }}(\theta)$ for the three fields, we follow the analysis described in Brown et al. (2003). We split each field into eight chip-sized sections and calculate the relevant correlation function $\left(c_{\mathrm{i}, \mathrm{s}}\right)$ for each section,

$c_{\mathrm{i}, \mathrm{s}}(\theta)=\frac{\sum_{\mathrm{ab}} W_{\mathrm{ab}} \gamma\left(\boldsymbol{\theta}_{a}\right) \gamma^{*}\left(\boldsymbol{\theta}_{b}\right)}{\sum_{\mathrm{ab}} W_{\mathrm{ab}}}$,

where $W_{\mathrm{ab}}$ is the weight for the galaxy pair $a b$. For the distant-pair data set $i=$ 'distant', $W_{\mathrm{ab}}=0$ if $\left|\hat{z}_{a}-\hat{z}_{b}\right|<\alpha \Delta_{z}$ and $W_{\mathrm{ab}}=1$ otherwise. For the close pairs $i=$ 'close' the weighting is simply reversed. Our estimate of the correlation function for the entire field is the average,

$C_{i}(\theta)=\frac{1}{N_{s}} \sum_{s=1}^{N_{s}} c_{i, s}(\theta)$,

where $N_{s}$ is the number of sections in a field. Note that here we are making the approximation that sections of the same field are uncorrelated. Neighbouring sections of the same field are, in fact, correlated but our approximation will be valid on scales which are small compared to the size of the sections (=8 $\times 16$ arcmin). With this approximation, an estimate of the covariance matrix of the $C_{i}(\theta)$ measurements is given by

$$
\begin{aligned}
& \operatorname{cov}\left[C_{i}(\theta) C_{j}\left(\theta^{\prime}\right)\right] \simeq \\
& \quad \frac{1}{N_{s}^{2}} \sum_{s=1}^{N_{s}}\left[c_{i, s}(\theta)-C_{i}(\theta)\right]\left[c_{j, s}\left(\theta^{\prime}\right)-C_{j}\left(\theta^{\prime}\right)\right] .
\end{aligned}
$$

We have investigated the sensitivity of the results to the choice of the number of sections each field is divided into, considering 4,8 and 16 sections. The most probable solution for $\sigma_{8}\left(\Omega_{m} / 0.27\right)^{0.6}$ varies by about 0.03 , much less than the statistical error, which is 


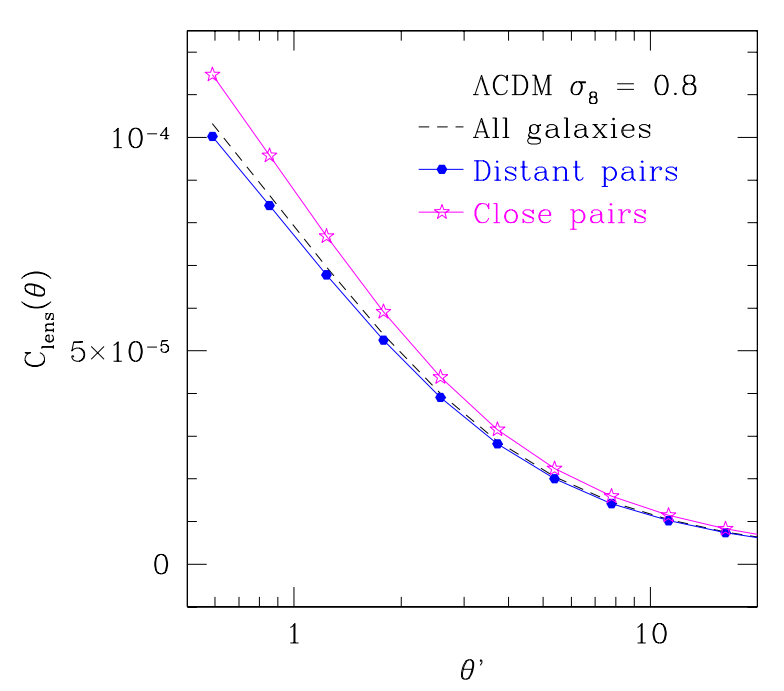

Figure 7. Predictions for the weak lensing shear correlation functions for the distant pairs and for the close pairs as described in the text. The difference in amplitude between the predicted signals is $\Delta C_{\text {lens }}(\theta) \leq$ $1.5 \times 10^{-5}$ at all measured scales.

about 0.1 (see Section 6). We do, however, add this error in quadrature in the quoted results.

\subsection{An observationally constrained intrinsic alignment model}

We have applied equations (29), (31) and (32) to the three fields, A901, CDFS and S11 and have combined the resulting measurements with minimum variance weighting to yield our final measurements of the intrinsic alignment signal for the close pairs in the COMBO-17 dataset. These final measurements are shown in Fig. 8 along with the predicted intrinsic alignment signal for the close pairs,

$C_{I A}(\theta)=\frac{\int d z_{a} d z_{b} \phi_{z}\left(z_{a}\right) \phi_{z}\left(z_{b}\right)\left[1+\xi_{g g}\right]\left\langle W_{a b}\right\rangle \eta\left(r_{a b}\right)}{\int d z_{a} d z_{b} \phi_{z}\left(z_{a}\right) \phi_{z}\left(z_{b}\right)\left[1+\xi_{g g}\right]\left\langle W_{a b}\right\rangle}$

where we use three different models for the intrinsic alignment $\eta\left(r_{a b}\right)$, and $\left\langle W_{a b}\right\rangle$ is given by

$\left\langle W_{a b}\right\rangle=\frac{1}{2 \sqrt{\pi}} \int_{-\infty}^{\infty} d x e^{-x^{2}}[\operatorname{erf}(y+x)-\operatorname{erf}(v+x)]$

where,

$x \equiv \frac{\hat{z}_{a}-z_{a}}{\sqrt{2} \Delta_{z}} \quad y \equiv \frac{z_{a}-z_{b}+\alpha \Delta_{z}}{\sqrt{2} \Delta_{z}} v \equiv \frac{z_{a}-z_{b}-\alpha \Delta_{z}}{\sqrt{2} \Delta_{z}}$.

(Heymans \& Heavens 2003). Fig. 9 shows the correlation matrix of the $C_{\mathrm{IA}}(\theta)$ measurements, defined by

$\operatorname{cor}\left(\theta, \theta^{\prime}\right)=\frac{\operatorname{cov}\left(\theta, \theta^{\prime}\right)}{\sqrt{\operatorname{cov}(\theta, \theta) \operatorname{cov}\left(\theta^{\prime}, \theta^{\prime}\right)}}$,

where $\operatorname{cov}\left(\theta, \theta^{\prime}\right)=\operatorname{cov}\left[C_{\mathrm{IA}}(\theta), C_{\mathrm{IA}}\left(\theta^{\prime}\right)\right]$ is the covariance matrix of the $C_{\mathrm{IA}}(\theta)$ measurements, which we estimate from the data using equations (32) and (29). One can immediately see from Fig. 8, in agreement with the aperture mass $\mathrm{B}$ mode analysis in Section 3 , that the measured COMBO-17 $C_{\mathrm{IA}}(\theta)$ strongly rejects the Jing model for the intrinsic alignment signal while the HRH model is

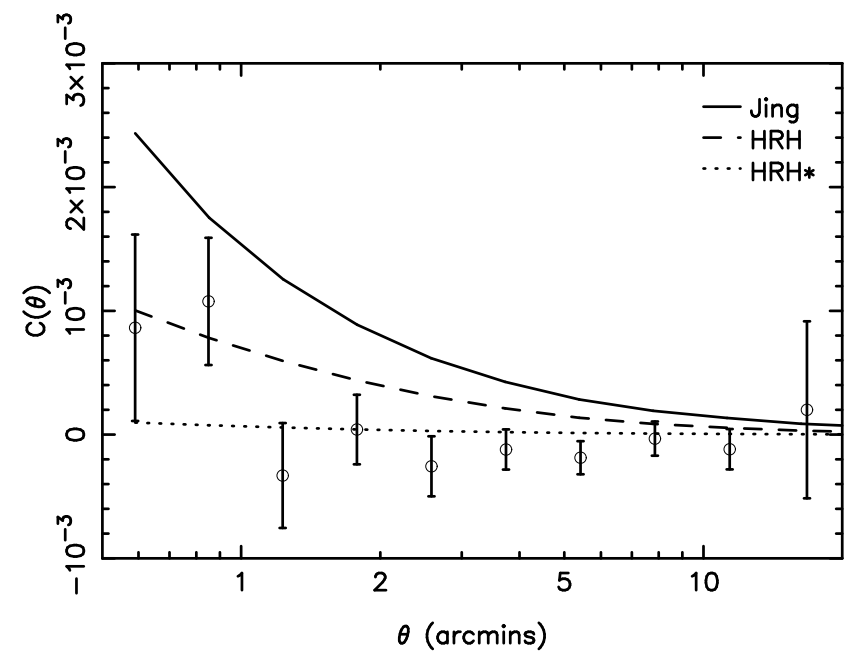

Figure 8. The intrinsic shear correlation functions from close galaxy pairs in the COMBO-17 survey, compared to predictions from Jing (solid), HRH (dashed) and $\mathrm{HRH}^{*}$ (dotted). The CNPT prediction lies slightly above the $\mathrm{HRH}^{*}$ prediction but on this scaling is indistinguishable and is therefore not plotted.

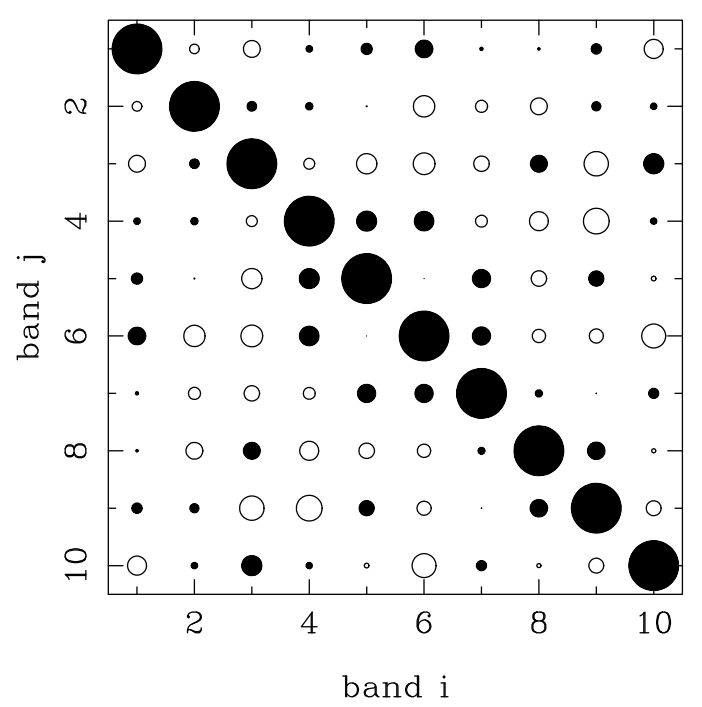

Figure 9. Correlation matrix of the optimally combined $C_{\mathrm{IA}}(\theta)$ measurements plotted in Fig. 8. The area of each circle is proportional to the degree of correlation between points $i$ and $j$. Filled circles denote that the points are correlated whereas unfilled circles denote an anti-correlation between the points. The points are the same as those plotted in Fig. 8, numbered 1 to 10 , in order from left to right.

also highly inconsistent with the data. The modified HRH model, $\mathrm{HRH}^{*}$, introduced in Section 2.2, and therefore also the CNPT model, are much better fits to the data. We note also that the $C_{\mathrm{IA}}(\theta)$ measurements are consistent with a null result on scales $>1$ arcmin although, from Fig. 9 it's clear that there are some (anti-) correlations between the correlation function measurements at different scales.

We calculate the likelihood of our $\mathrm{HRH}^{*}$ model from the COMBO-17 results allowing the amplitude to vary, see Fig. 10. We find that the best fit is at zero amplitude, and that an $\mathrm{HRH}^{*}$ model with an amplitude greater than $A=0.0054$ can be rejected with 


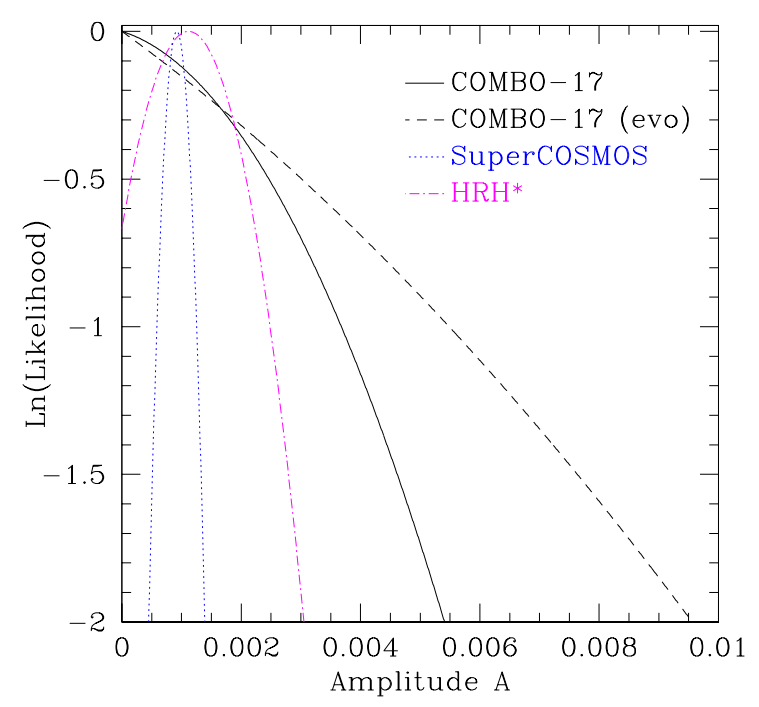

Figure 10. The likelihood of the amplitude A of the model fit for the intrinsic galaxy alignment correlation function equation (13), as determined from the COMBO-17 photometric redshift dataset assuming no evolution galaxy clustering, (solid), and assuming stable galaxy clustering, (dashed), see Section 5.2. Over-plotted is the likelihood of the amplitude from the modified $\mathrm{HRH}^{*}$ results, (dot dash), and the likelihood of the amplitude from the SuperCOSMOS ellipticity variance measurements, (dotted), as detailed in Section 2.2.

95\% confidence. We therefore find an observationally constrained maximum amplitude intrinsic alignment model such that:

$\eta_{\mathrm{C} 17}(r)<\frac{0.0054}{1+r^{2}} \quad 95 \%$ confidence.

Note that using $16 \times 16$ arcminute sections in the correlation estimator equation (31), we find consistent results with a $95 \%$ upper limit $A=0.0039$.

\subsection{Application of the HH weighting scheme to COMBO-17}

With our constraints on the amplitude of the intrinsic alignment signal from the previous section, we can now apply the HH weighting scheme to the COMBO-17 data. In order to compare with the expected $\Lambda C D M$ weak lensing shear signal, we apply the weighting scheme to the CDFS and S11 fields only, in order to avoid the much larger shear signal seen in the A901 field due to the presence of the supercluster (Brown et al. 2003). To calculate the optimal $\alpha(\theta)$ weights, (see appendix), we use the COMBO-17 redshift distribution for the CDFS and S11 fields, with our chosen input model for the intrinsic alignment signal as given by the upper limit of $\eta_{\mathrm{C} 17}(r)$ equation (37), thereby ensuring removal of any feasible intrinsic alignment contamination. With values for $\alpha(\theta)$, shown in Fig. A2, we calculate the total and distant-pair correlation functions as given by equations (30) and (31), the results of which are shown in Fig. 11. The total $C(\theta)$ (circles) will include intrinsic alignment contamination, whereas the distant-pair signal (triangles) excludes virtually all intrinsic alignment at the expense of a small increase in the shot noise. Over-plotted is the cosmic shear prediction for a $\Lambda$ CDM model, equation (25), normalised to $\sigma_{8}=0.8$, where the COMBO-17 photometric redshift distribution for the CDFS and S11 fields has been used for this calculation.

This result is a good example of the application of the $\mathrm{HH}$

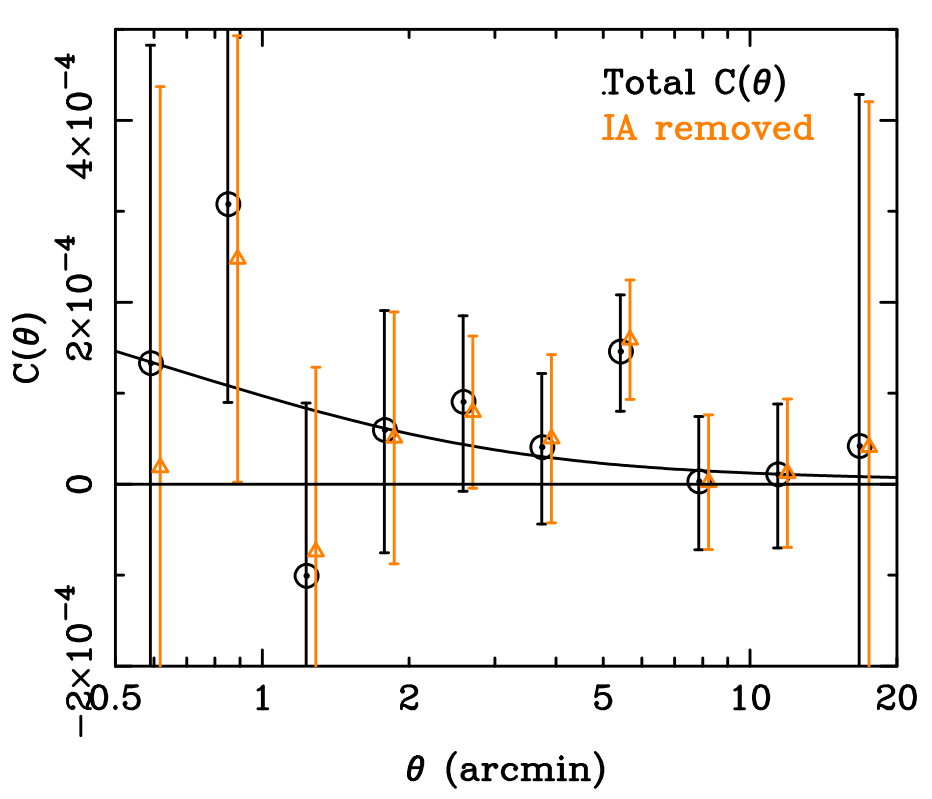

Figure 11. Application of the intrinsic alignment suppression weighting scheme as described in the text. The circles are the total correlation function $C(\theta)$ measurements from all galaxy pairs whereas the triangles are the $C(\theta)$ measurements from just the distant galaxy pairs. The curve plotted is the expected weak lensing signal for a $\Lambda$ CDM model normalised to $\sigma_{8}=0.8$.

weighting to a weak lensing data set, effectively removing the systematic error from intrinsic galaxy alignments whilst producing only a small increase in the shot noise. Due to the fact that we are only using a small subset of the COMBO-17 data set (0.5 square degrees limited to magnitude $R<24$ ), we are unable to use this noisy result directly to investigate the effect on cosmological parameter estimation. We can, however, use the likelihood of the amplitude of the intrinsic alignment signal to calculate its effect on parameter estimation from the full COMBO-17 deep $R$-band sample. We do this in Section 6.

\section{EFFECT OF GALAXY CLUSTERING EVOLUTION}

All previous studies of intrinsic galaxy alignment have assumed weak evolution in galaxy clustering which becomes important when converting measured 3D intrinsic ellipticity correlations, $\eta(r)$, into angular correlation functions $C_{I}(\theta)$, see equation (5). If there is evolution in galaxy clustering such that at high redshift there are proportionally less nearby galaxy pairs than at low redshifts, this will lower predictions of $C_{I}(\theta)$. The clustering evolution of galaxies has often been quantified, although without strong theoretical motivation, by a redshift-dependent two point correlation function of the form (Groth \& Peebles 1997)

$\xi_{g g}(r, z)=\left(\frac{r}{r_{0}}\right)^{\gamma}(1+z)^{-(3+\epsilon)}$.

Observational studies have found that this simple redshiftdependent model provides a poor fit to data (Wilson 2003; McCracken et al. 2001), which is most likely due to the fact that different populations of galaxies have different intrinsic clustering. Parameters for equation (38) are therefore observationally challeng- 
ing to determine and as yet fairly uncertain. Wilson (2003) investigates the redshift evolution for a single population of galaxies in the UH8K weak lensing fields and it is these results that we will use in order to investigate the effects of galaxy clustering evolution in intrinsic alignment studies such that $\gamma=-1.8, r_{0}=5.25 h^{-1} \mathrm{Mpc}$ and $\epsilon=0$. For $\epsilon=0$, the evolution is of stable clustering, where the galaxies are dynamically bound and stable at small scales.

Fig. 12 shows the predicted angular shear correlation function, $\left\langle\gamma \gamma^{*}\right\rangle_{I}=\frac{1}{4} C_{I}(\theta)$ calculated including galaxy clustering evolution for each intrinsic alignment model for the RCS and VIRMOSDESCART surveys. We also plot for comparison the shear correlations expected from weak gravitational lensing, equation (25). Comparing this with Fig. 5 we note that by assuming stable clustering evolution, the intrinsic alignment contribution at small angular scales is significantly reduced compared to previous results. This is due to the fact that it is at small angular scales that high redshift galaxy pairs are close enough to contribute to any intrinsic correlations. If galaxies are less strongly clustered at high redshift then there will be fewer high-redshift close pairs and hence the angular intrinsic correlation function is reduced.

\subsection{Aperture mass B modes}

We repeat the analysis of Section 3 with the intrinsic alignment correlation functions now calculated including galaxy clustering evolution, as shown in Fig. 12. Fig. 13 shows these results, comparing the aperture mass B mode measurements reported by the RCS, (upper panel), and VIRMOS-DESCART survey, (lower panel), and the four different intrinsic alignment B modes. Unlike the clear result from Section 3 the inclusion of stable galaxy clustering reduces the expected B mode contribution from intrinsic galaxy alignments, permitting all intrinsic alignment models where the observed B modes are considered as upper limits.

\subsection{COMBO-17 Correlation Analysis}

We have also investigated the effect of including galaxy evolution in the intrinsic alignment models for the COMBO-17 correlation analysis. The resulting $C_{\mathrm{IA}}(\theta)$ measurements are shown in Fig. 14 along with the predictions for the Jing, HRH and $\mathrm{HRH}^{*}$ intrinsic alignment models where stable clustering galaxy evolution has been included. Here, we find that, although not as clear a result as found in Section 4, we can still exclude the Jing model and, once again, the $\mathrm{HRH}^{*}$ model is favoured. We calculate the likelihood of our $\mathrm{HRH}^{*}$ model which includes clustering evolution, allowing the amplitude A to vary, see Fig. 10. We find again that the best fit is at zero amplitude, and that an $\mathrm{HRH}^{*}$ evolving model with an amplitude greater than $A=0.0096$ can be rejected with $95 \%$ confidence.

\section{IMPLICATIONS FOR WEAK LENSING MEASUREMENTS}

\subsection{Cosmological parameter constraints}

We now turn to the implications of our intrinsic alignment amplitude constraints for cosmological parameter estimation. To do this we follow Brown et al. (2003) using measurements from the deep $R<25.5$ COMBO-17 data of the two correlation functions, $C_{1}(\theta)=E\left[\gamma_{t} \gamma_{t}: \theta\right]$ and $C_{2}(\theta)=E\left[\gamma_{r} \gamma_{r}: \theta\right]$ from equation (31), with $W_{a b}=1$ for all galaxy pairs, in order to obtain a joint
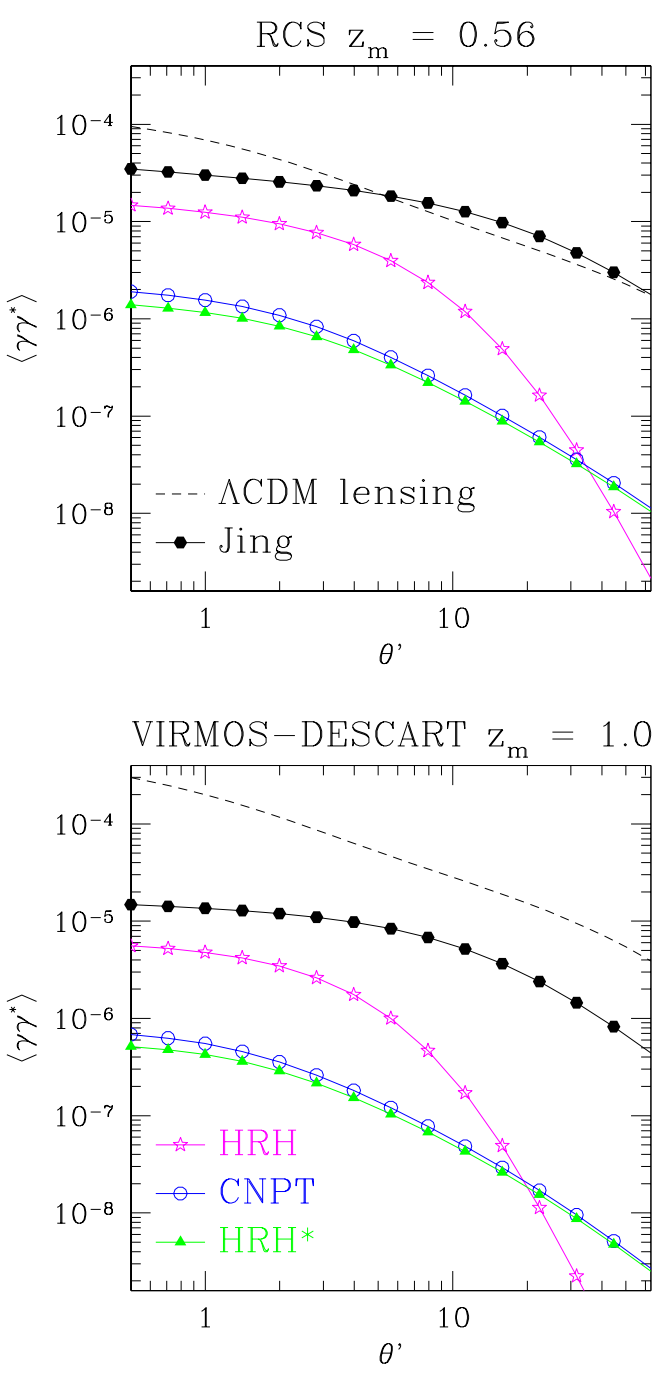

Figure 12. Predicted intrinsic alignment angular shear correlation functions $\left\langle\gamma \gamma^{*}\right\rangle_{I}$ including stable galaxy clustering evolution, Jing (filled), HRH (stars), CNPT (circles) and HRH* (triangles). The intrinsic alignment contributions are compared to the angular shear correlations from $\Lambda$ CDM weak gravitational lensing, normalised to $\sigma_{8}=0.8$, (dashed), for the RCS with depth $z_{m}=0.56$, (upper) and for the VIRMOS-DESCART survey with depth $z_{m}=1.0$, (lower).

measurement of the normalisation of the matter power spectrum, $\sigma_{8}$ and the matter density, $\Omega_{m}$. We perform a $\chi^{2}$ fitting procedure on the correlation function measurements ordered in a data vector $\mathbf{d}=\left\{C_{1}\left(\theta_{1}\right), \ldots, C_{1}\left(\theta_{n}\right), C_{2}\left(\theta_{1}\right), \ldots, C_{2}\left(\theta_{n}\right)\right\}$, for a set of theoretical parameters, $\left(\sigma_{8}, \Omega_{m}, H_{0}, A\right)$, where $H_{0}$ is the Hubble parameter and $\mathrm{A}$ is the amplitude of the intrinsic alignment signal in equation (13), by calculating

$\chi^{2}=\left[\mathbf{d}-\mathbf{x}\left(\sigma_{8}, \Omega_{m}, H_{0}, A\right)\right]^{T} \mathbf{V}^{-1}\left[\mathbf{d}-\mathbf{x}\left(\sigma_{8}, \Omega_{m}, H_{0}, A\right)\right]$,

where $\mathbf{x}\left(\sigma_{8}, \Omega_{m}, H_{0}, A\right)$ is a theory vector containing the $C_{1}(\theta)$ and $C_{2}(\theta)$ correlation functions calculated for the cosmological model. $\mathbf{V}=\left\langle\mathbf{d d}^{T}\right\rangle$ is the covariance matrix of the data measurements which can be estimated, assuming that sections of the same field are uncorrelated, from equation 32 . We assume a flat cosmology $\Omega_{m}+\Omega_{\Lambda}=1$ and a nonlinear CDM matter power spectrum, $P_{\delta}$, calculated using the fitting formula from Smith et al. (2003) with the transfer function of Eisenstein \& Hu (1999), where the 

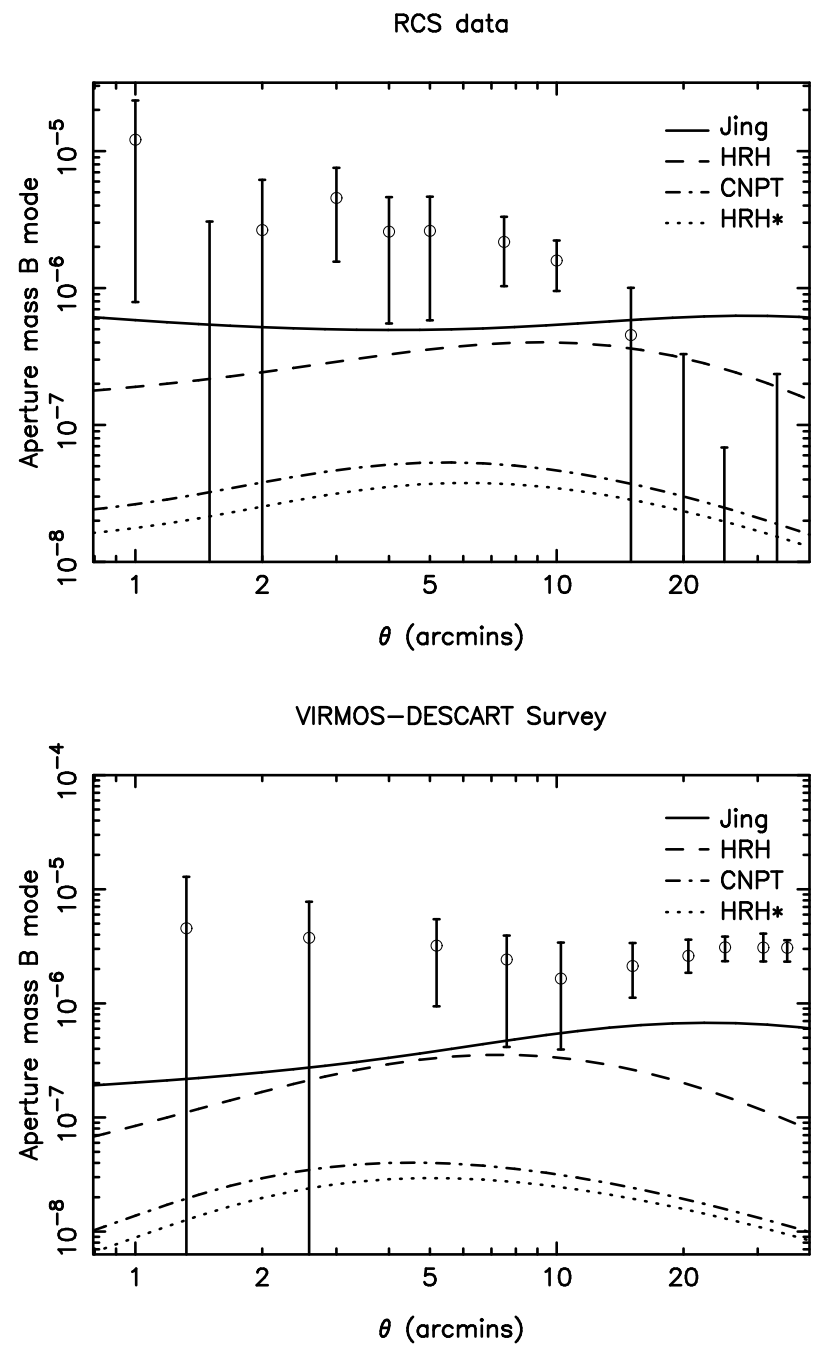

Figure 13. Intrinsic alignment contributions to the aperture mass B mode $\left\langle M_{\perp}^{2}\right\rangle$ assuming evolution in galaxy clustering; Jing(solid), HRH (dashed) and CNPT(dot dash). The upper panel compares stable clustering intrinsic alignment predictions for the RCS survey with their measured B mode. The lower panel compares stable clustering intrinsic alignment predictions for the VIRMOS-DESCART with their measured B mode.

initial slope of the power spectrum is $n=1$. In contrast to (Brown et al. 2003), we do not fix the Hubble constant $H_{0}$, but marginalise over it, with a Gaussian prior $p\left(H_{0}\right)$ set by the WMAP results with $H_{0}=72 \pm 5 \mathrm{~km} \mathrm{~s}^{-1} \mathrm{Mpc}^{-1}$ (Spergel et al. 2003).

We calculate $\chi^{2}$ equation (39), for values of $\sigma_{8}$ ranging from 0.3 to 1.5 and values of $\Omega_{m}$ ranging from 0.1 to 1.0 , on a grid with 0.05 spacing in both these parameters, for two cases (a) the case where we ignore any effect of intrinsic alignments setting $A=0$ and (b) the case where we also vary values of $A$ from 0.0 to 0.01 in steps of 0.0005 and then marginalise over the intrinsic alignment amplitude. For the marginalisation we use the probability distribution for the intrinsic alignment amplitude $p(A)$ obtained from COMBO-17 and shown in Fig. 10 such that

$p\left(\sigma_{8}, \Omega_{m}\right)=\int d A \int d H_{0} p\left(\sigma_{8}, \Omega_{m}, H_{0}, A\right) p\left(H_{0}\right) p(A)$

where $p\left(\sigma_{8}, \Omega_{m}, H_{0}, A\right) \propto \exp \left(-\chi^{2} / 2\right)$. As in Section 3, we assume that intrinsic galaxy alignments have no preferred tangential

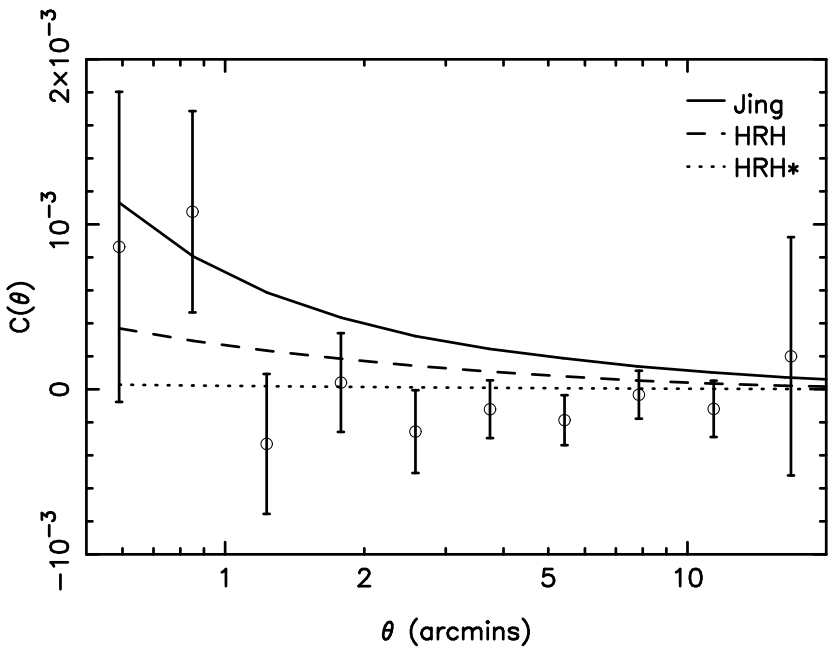

Figure 14. The intrinsic shear correlation function from close galaxy pairs in the COMBO-17 survey, compared to predictions from Jing (solid), HRH (dashed) and $\mathrm{HRH}^{*}$ (dotted) for stable clustering galaxy evolution.

or radial alignment, and that $C_{\mathrm{I}}(\theta)_{t t} \approx C_{\mathrm{I}}(\theta)_{r r} \approx \frac{1}{2} C_{\mathrm{I}}(\theta)$, where $C_{\mathrm{I}}(\theta)$ is now calculated for the deep $R<25.5$ COMBO-17 estimated redshift distribution detailed in Brown et al. (2003).

The resulting constraints in the $\sigma_{8}-\Omega_{m}$ plane are shown in Fig. 15. Assuming no intrinsic alignment signal, we obtain a best fit measurement for the normalisation of the power spectrum of

$\sigma_{8}\left(\Omega_{m} / 0.27\right)^{0.6}=0.74 \pm 0.11$,

while for the case where we have marginalised over the intrinsic alignment amplitude derived assuming no clustering evolution, we find our estimate drops to

$\sigma_{8}\left(\Omega_{m} / 0.27\right)^{0.6}=0.71 \pm 0.11$.

The errors are larger than quoted in Brown et al. (2003) because we have marginalised over $H_{0}$ rather than fixing it at 68 $\mathrm{km} \mathrm{s}^{-1} \mathrm{Mpc}^{-1}$, but they remain relatively small in comparison to other surveys, bearing in mind the COMBO-17 survey area. This is a result of the good determination of source redshifts in the COMBO-17 survey, and the relatively high number density of resolved background sources used in this analysis. If we marginalise over the $H_{0}$ distribution obtained from $\mathrm{CMB}$ and $2 \mathrm{dF}$ with $H_{0}=$ $68 \pm 5 \mathrm{~km} \mathrm{~s}^{-1} \mathrm{Mpc}^{-1}$ (Efstathiou et al. 2002; Percival et al. 2002), these numbers change to $\sigma_{8}\left(\Omega_{m} / 0.27\right)^{0.6}=0.78$ and 0.77 , with an error of 0.12 . Note that all these error bars include a small error added in quadrature from the imperfect estimation of the covariance matrix (section 4.1).

Note that if we instead use measurements of the total correlation function, $C(\theta)=C_{1}(\theta)+C_{2}(\theta)$ we find the same reduction of 0.03 in our estimates of $\sigma_{8}$. With the intrinsic alignment constraints from the SuperCOSMOS survey we find a reduction in our estimates of $\sigma_{8}$ of 0.01 . Fig. 16 shows the COMBO-17 measurement of the shear correlation function along with the predicted lensing shear correlation function with the HRH* intrinsic alignment signal subtracted.

For the case of stable clustering galaxy evolution, the resulting angular intrinsic alignment contamination significantly decreases, and we find that the constraints in the $\sigma_{8}-\Omega_{m}$ plane are unchanged after marginalising over the intrinsic alignment signal, derived assuming stable clustering galaxy evolution. 


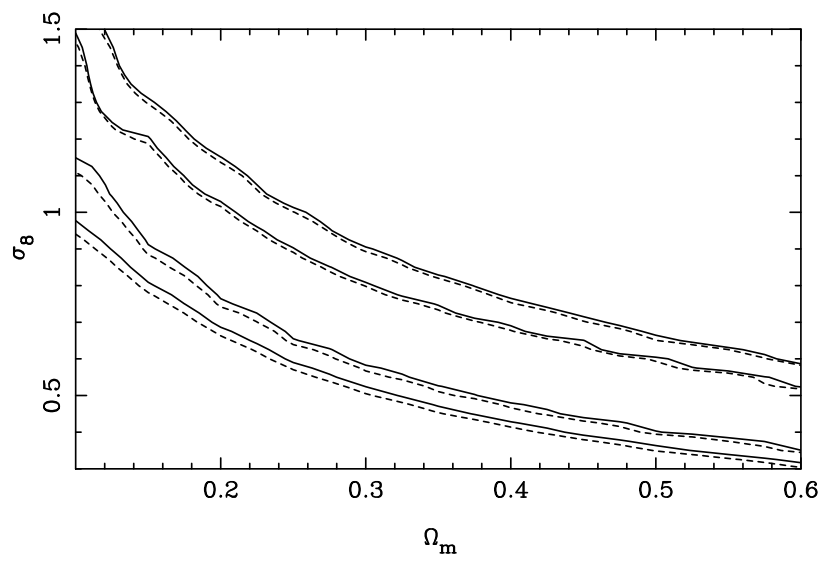

Figure 15. The probability surface for $\sigma_{8}$ and $\Omega_{m}$ from COMBO-17 as calculated using the shear correlation function measurements $C_{1}(\theta)$ and $C_{2}(\theta)$ in combination, after marginalising over the Hubble constant. The intrinsic alignment signal is either assumed to be zero (solid contours) or is marginalised over (dashed contours). The inner and outer contours in each case correspond to $\Delta \chi^{2}=2.3$ and 6.17 . confidence regions respectively.

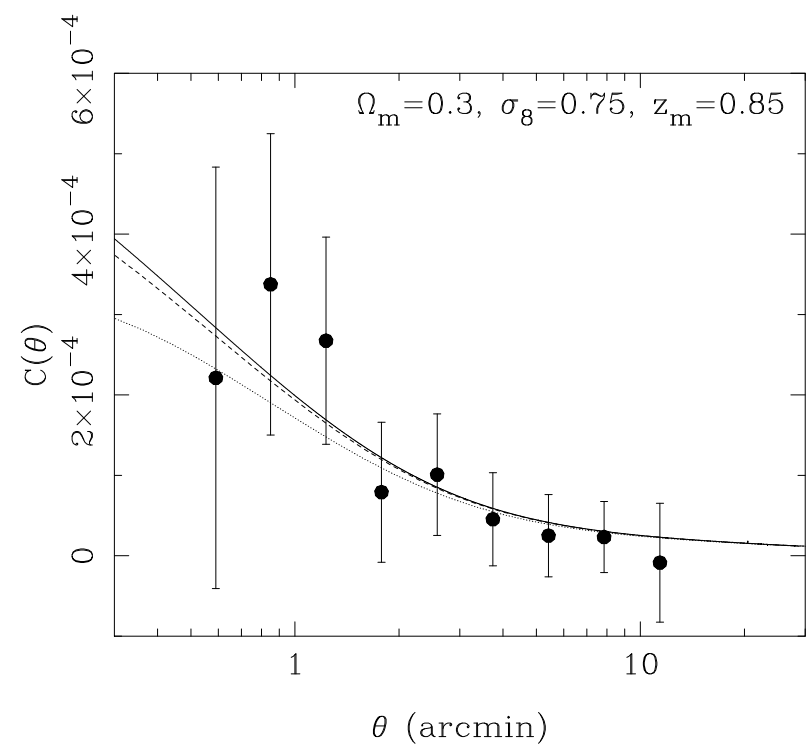

Figure 16. The total shear correlation function for the deep COMBO-17 survey, along with the best fit $\Lambda \mathrm{CDM}$ weak lensing correlation function assuming no intrinsic alignments (solid). Subtracting off the the best-fitting intrinsic alignment model $\left(\mathrm{HRH}^{*}\right)$ decreases the lensing correlation function to the dashed line, and with the $95 \%$ upper limit (obtained from the shallower survey with photometric redshifts) the lensing correlation function decreases to the dotted line.

\subsection{Implications for future surveys : CFHTLS and SNAP}

The implications for weak lensing surveys is obviously dependent, as shown in Section 5, on our understanding of galaxy clustering evolution. Until agreement is reached upon the true redshift dependence of galaxy clustering we consider the contamination to weak lensing measurements derived assuming no evolution in galaxy clustering, which should be considered as upper limits as redshift evolution will dilute the signal. For the modified HRH model $\eta_{\mathrm{HRH} *}(r)$ we find that

$\frac{C_{I}}{4\left\langle\gamma \gamma^{*}\right\rangle}(\theta=1 \operatorname{arcmin})=0.02(\mathrm{CFHTLS})$

$$
=0.01(\mathrm{SNAP}) \text {, }
$$

where we have calculated $C_{I}(\theta)$ from equation (5) with $\phi_{z}(z)$ as given in equation (6), with $z_{m}=1.0$ for the CFHTLS wide survey, and $z_{m}=1.23$ for the SNAP wide survey. $\left\langle\gamma \gamma^{*}\right\rangle$ has been calculated from equation (25) with $\Omega_{m}=0.3, \Omega_{\Lambda}=0.7, \sigma_{8}=0.8$ and $\Gamma=0.2$. The contamination of weak lensing measurements at angular scales $\theta>1$ arcmin is therefore less than $2 \%$ for CFHTLS, and less than $1 \%$ for SNAP. These estimates are essentially unchanged if we use the SuperCOSMOS measurement of intrinsic alignments. Using the $95 \%$ upper limit for the intrinsic alignment amplitude $\eta_{\mathrm{C} 17}(r)$, obtained from the COMBO-17 data, these estimates are multiplied by approximately 5 , giving the $95 \%$ upper limits on any possible intrinsic alignment contamination at $10 \%$ for CFHTLS and 5\% for SNAP. For comparison, with stable clustering evolution, contamination for both surveys is practically negligible, less that $0.2 \%$ for CFHTLS, and less that $0.1 \%$ for SNAP.

Refregier et al. (2003) have shown that for SNAP, the application of redshift tomography as proposed by $\mathrm{Hu}$ (1999), increases the accuracy of cosmological parameter estimates by a factor of about 2 . This method involves splitting the galaxy sample into two or three redshift slices, and is therefore more susceptible to higher levels of intrinsic alignment contamination (Croft \& Metzler 2000). Using the redshift slice distributions as proposed by Refregier et al. (2003), we have calculated the intrinsic alignment contamination for the best-fit $\mathrm{HRH}^{*}$ model, and found that for the three SNAP redshift slices with median redshifts $0.8,1.3$ and 1.9 , the contamination at angular scales $\theta>1$ arcmin is found to be, $7.0 \%, 2.5 \%$ and $0.7 \%$ respectively. For the lowest redshift bin, this level of contamination is significant, especially if we conservatively consider the 95\% upper limit from our COMBO-17 constraint, which increases the contamination by a factor of approximately 5 to $\sim 35 \%$. It will therefore be important, when using tomography in weak lensing analysis, to apply a close galaxy pair downweighting scheme in order to remove this systematic error. Note that when considering stable galaxy clustering evolution the contamination is $1 \%$ for the lowest SNAP redshift bin.

\section{CONCLUSIONS}

The weak correlation of the ellipticities of galaxy images is an indicator of gravitational lensing and a powerful tool for the study of dark matter on large scales. In this paper we have used two methods to estimate the extent to which this statistic is contaminated by the intrinsic physical alignments of galaxies. Our main conclusion is that the effect is relatively small, but not entirely negligible, and, for the COMBO-17 survey, leads to a reduction in the derived amplitude of mass clustering of around $3 \%$.

For the brighter part $(R<24)$ of the COMBO-17 survey, which has photometric redshifts, we removed close pairs from the shear correlation analysis, removing the intrinsic alignment signal, as described by Heymans \& Heavens (2003). Comparing this shear correlation function from the distant pairs with that of the close pairs then allows us to estimate the intrinsic alignment signal. We have also placed limits on the intrinsic alignment signal from analysis of the aperture mass B mode in the RCS and VIRMOSDESCART surveys. We find a consistent picture that this signal is lower than that expected from analysis of numerical simulations (Heavens, Refregier \& Heymans 2000; Jing 2002), but in broad agreement with the semi-analytic calculation of Crittenden et al. (2002).

We have reanalysed the numerical simulations to include two 
effects which were originally ignored. These are a misalignment between the angular momentum of the baryons and the halo (van den Bosch et al. 2002), and the finite thickness of disk galaxies (Crittenden et al. 2002). Both these effects reduce the intrinsic galaxy ellipticity correlation function to a level similar to that found by Crittenden et al. (2002), and consistent with the level determined observationally in this paper and the level measured in the SuperCOSMOS survey (Brown et al. 2002). Note that other effects such as gas-dynamical interaction have not been taken into account, which could cause the intrinsic alignment signal to be lowered still further.

Having estimated the contribution of intrinsic alignments to the brighter part of the COMBO-17 data, we have computed the likelihood for the contamination of the whole COMBO-17 sample which extends to $R<25.5$. We compute the probability distribution for $\sigma_{8}\left(\Omega_{m} / 0.27\right)^{0.6}$, now marginalising over the Hubble constant. Marginalising over the amplitude of the intrinsic alignment contamination, the mass clustering amplitude $\sigma_{8}\left(\Omega_{m} / 0.27\right)^{0.6}=$ $0.71 \pm 0.11$. Ignoring intrinsic alignments leads to a systematic overestimate by 0.03 .

From the COMBO-17 results we have also calculated $95 \%$ upper limits for the expected contamination of future surveys, CFHTLS $(<10.0 \%)$ and SNAP $(<5 \%)$. With our theoretical model, or with the intrinsic alignment signal estimated from the SuperCOSMOS data, these predicted limits become $2 \%$ and $1 \%$ respectively. With current surveys these levels of contamination are small enough to be neglected, but will be significant in the error budget of future high-precision weak lensing surveys. Both CFHTLS and SNAP aim to produce accurate photometric redshift estimates for their galaxy sample enabling the use of redshift tomography to further improve cosmological parameter estimation. This technique is susceptible to significant contamination from intrinsic galaxy alignments due to the thin widths of the redshift bins, increasing the proportion of nearby galaxy pairs. We have shown that, even with the low amplitude intrinsic alignment model $\eta_{\mathrm{HRH} *}(r)$, with the proposed redshift distributions for the SNAP tomographic redshift bins, the lowest redshift bin will suffer contamination $\sim 7 \%$, with a $95 \%$ upper limit of $35 \%$ if we consider our observationally constrained upper limits for $\eta_{\mathrm{C} 17}(r)$. Since these surveys will have photometric redshift information, it will therefore be vital to remove the intrinsic alignment signal using the exclusion of nearby galaxy pairs as proposed by Heymans \& Heavens (2003) and King \& Schneider (2002).

Our conclusions are affected by the clustering strength of galaxies, as this partly determines how many pairs of galaxies which are close on the sky are actually physically close together, and susceptible to physical interactions which could lead to intrinsic alignments. The results we have presented so far assume that clustering is independent of redshift, but for illustration we have also investigated, without strong theoretical motivation, an evolutionary model corresponding to stable galaxy clustering. In this case, the effects at high redshift are reduced to a negligible level for COMBO-17, CFHTLS and SNAP, but could still be important in the case of weak lensing tomography analysis.

In the process of applying weighting schemes as proposed by Heymans \& Heavens (2003) and King \& Schneider (2002) to future weak lensing surveys, there will potentially be some very interesting by-products. For example, with large area redshift slices, the method detailed in Section 4.1 could be applied in order to determine the strengths of intrinsic galaxy alignments as a function of redshift, which throughout this paper we have assumed to be constant. In principle this could be a useful constraint for galaxy for- mation and evolution studies. With the SuperCOSMOS results and our COMBO-17 intrinsic alignment constraint favouring an intrinsic alignment model which includes misalignments between baryon and halo angular momentum, there is now observational evidence indirectly supporting the finding by van den Bosch et al. (2002), which has important implications for formation of disk galaxies. We therefore conclude that although, for studies of weak gravitational lensing, the presence of intrinsic galaxy alignments is an inconvenience, they are an interesting topic in their own right.

\section{ACKNOWLEDGEMENTS}

MLB thanks the University of Edinburgh for support during the writing of this paper. $\mathrm{CW}$ was supported by the PPARC rolling grant in Observational Cosmology at University of Oxford. The simulations analysed in this paper were carried out using data made available by the Virgo Supercomputing Consortium (starwww.dur.ac.uk/frazerp/virgo/) using computers based at the Computing centre of the Max-Planck Society in Garching at the at Edinburgh Parallel Computing Centre. We are very grateful to Rob Smith for providing us with halos from the simulations and his non-linear power spectrum fitting formula and code. We thank Andi Burkert, Henk Hoekstra, Paul Allen, Priya Natarajan, Rob Crittenden and Stephi Phleps for useful discussions, and the referee for very helpful comments.

\section{REFERENCES}

Adelberger K., Steidel C. G., Giavalisco M., Dickinson M., Pettini M., Kellogg M., 1998. ApJ, 505, 18.

Bacon D., Taylor A., 2003. MNRAS, 344, 1307.

Bacon D., Massey R., Refregier A., Ellis R., 2003. MNRAS, 344, 673.

Bacon D., Refregier A., Ellis R., 2000. MNRAS, 318, 625.

Bartelmann M., Schneider P., 2001. Physics Reports, 340, 291.

Baugh C., Benson A., Cole S., Frenk C., Lacey C., 1999. MNRAS, 305, L21.

Benabed K., Van Waerbeke L., 2003. Phys. Rev. D, submitted, astroph/0306033,

Brown M., Taylor A., Hambly N., Dye S., 2002. MNRAS, 333, 501.

Brown M., Taylor A., Bacon D., Gray M., Dye S., Meisenheimer K., Wolf C., 2003. MNRAS, 341, 100.

Carlberg R. G., Yee H. K. C., Morris S. L., Lin H., Hall P. B., Patton D., Sawicki M., Shepherd C. W., 2000. ApJ, 542, 57.

Catelan P., Kamionkowski M., Blandford R. D., 2001. MNRAS, 320, L7.

Cole S., Ellis R., Broadhurst T., Colless M., 1994. MNRAS, 267, 541.

Contaldi C., Hoekstra H., Lewis A., 2003. Phys. Rev. Lett., 90, 303.

Crittenden R., Natarajan R., Pen U., Theuns T., 2001. ApJ, 559, 552.

Crittenden R., Natarajan R., Pen U., Theuns T., 2002. ApJ, 568, 20.

Croft R. A. C., Metzler C. A., 2000. ApJ, 545, 561.

Ebbels T., 1998. PhD thesis, Cambridge University, .

Efstathiou G., Moody S., Peacock J. A., Percival W. J., Baugh C., Bland-Hawthorn J., Bridges T., Cannon R., Cole S., Colless M., Collins C., Couch, W. andDalton G., de Propris R., Driver S. P., Ellis R. S., Frenk C. S., Glazebrook K., Jackson C., Lahav, O. andLewis I., Lumsden S., Maddox 
S., Norberg P., Peterson B. A., Sutherland W., Taylor K., 2002. MNRAS, 330, L29.

Eisenstein D. J., Hu W., 1999. ApJ, 511, 5.

Firth A., Somerville R., McMahon R., Lahav O., Ellis R., Sabbey C., McCarthy P., Chen H., Marzke R., Wilson J., Abraham R., Beckett M., Carlberg R., Lewis J., Mackay C., Murphy D., Oemler A., Persson S., 2002. MNRAS, 332, 617.

Giavalisco M., Steidel C., Adelberge K., Dickinson M., Pettini M., Kellogg M., 1998. ApJ, 503, 543.

Groth E., Peebles J., 1997. ApJ, 217, 385.

Hamana T., Miyazaki S., Shimasaku K., Furusawa H., Doi M., Hamabe M., Imi K., Kimura M., Komiyama Y., Nakata F., Okada N., Okamura S., Ouchi M., Sekiguchi M., Yagi M., Yasuda N., 2002. ApJ, submitted, astroph/0210450, .

Heavens A., Refregier A., Heymans C., 2000. MNRAS, 319, 649.

Heavens A., 2003. MNRAS, submitted, astroph/0304151, .

Heymans C., Heavens A., 2003. MNRAS, 339, 711.

Hoekstra H., Yee H., Gladders M., Barrientos L. F., Hall P., Infante L., 2002. ApJ, 572, 55.

Hoekstra H., Yee H., Gladders M., 2001. ApJ, 558, L11.

Hoekstra H., Yee H., Gladders M., 2002. ApJ, 577, 595.

Hoekstra H., 2003. MNRAS, submitted, astroph/0306097, .

Hu W., Keeton C., 2002. Phys. Rev. D, 66, 3506.

Hu W., 1999. ApJ, 552, L21.

Hui L., Zhang J., 2002. ApJ, submitted, astroph/0205512, .

Jain B., Taylor A., 2003. Phys. Rev. Lett, submitted, astroph/0306046, .

Jarvis M., Bernstein G., Jain B., Fischer P., Smith D., Tyson J., Wittman D., 2003. ApJ, 125, 1014.

Jenkins A., Frenk C., Thomas P., Colberg J., White S., Couchman H., Peacock J., Efstathiou G., Nelson A., 1998. ApJ, 499, 20.

Jing Y. P., 2002. MNRAS, 335, L89.

Kaiser N., Squires G., Fahlman G., Woods D., 1994. Clusters of Galaxies, Editions Frontieres. Gif-sur-Yvette, , 269.

Kaiser N., Squires G., Broadhurst T., 1995. ApJ, 449, 460.

Keeton C. R., Kochanek C. S., Falco E. E., 1998. ApJ, 509, 561.

King L., Schneider P., 2002. A\&A, 396, 411.

King L., Schneider P., 2003. A\&A, 398, 23.

Lambas D. G., Maddox S. J., Loveday J., 1992. MNRAS, 258, 404.

Le Fevre O., Hudon D., Lilly S., Crampton D., Hammer F., Tresse L., 1996. ApJ, 461, 534.

Lee J., Pen U., 2001. ApJ, 555, 106.

Lee J., Pen U., Seljak U., 2002. ApJ, 543, L107.

Luppino G. A., Kaiser N., 1997. ApJ, 475, 20.

Mackey J., White M., Kamionkowski M., 2002. MNRAS, 332, 788.

Massey R., Rhodes J., Refregier A., Albert J., Bacon D., Bernstein G., Ellis R., Jain B., McKay T., Perlmutter S., Taylor A., 2003. ApJ submitted, astroph/0304418,

McCracken H. J., Le Fevre O., Brodwin M., Foucard S., S L., Crampton D., Mellier Y., 2001. A\& A, 376, 756.

Pen U., Lu T., Van Waerbeke L., Mellier Y., 2003. MNRAS submitted, astroph/0304512, .

Pen U.-L., Van Waerbeke L., Mellier Y., 2002. ApJ, 567, 31.

Percival W. J., Sutherland W., Peacock, J. A. andBaugh C. M., Bland-Hawthorn J., Bridges T., Cannon R., Cole S., Colless M., Collins C., Couch W., Dalton G., De Propris R., Driver S. P., Efstathiou G., Ellis R. S., Frenk C. S., Glazebrook K., Jackson C., Lahav O., Lewis I., Lumsden S., Maddox S., Moody S., Norberg P., Peterson B. A., Taylor K., 2002. MNRAS, 337, 1068.
Plionis M., Benoist C., Maurogordato S., Ferrari C., Basilakos S., 2003. ApJ, 594, 144.

Porciani C., Dekel A., Hoffman Y., 2002. MNRAS, 332, 325.

Postman M., Lauer T., Szapudi I., Oegerle W., 1998. ApJ, 506, 33. Refregier A., Massey R., Rhodes J., Ellis R., Albert J., Bacon D., Bernstein G., McKay T., Perlmutter S., 2003. ApJ submitted, astroph/0304419, .

Rhodes J., Refregier A., Massey R., Albert J., Bacon D., Bernstein G., Ellis R., McKay T., Akerlof C., Aldering G., Amanullah R., Astier P., Barrelet E., SNAP Collaboration, 2003. ApJ submitted, astroph/0304417, .

Rhodes J., Refregier A., Groth E. J., 2000. ApJ, 536, 79.

Rhodes J., Refregier A., Groth E., 2001. ApJ(Lett), 552, 85.

R.Maoli, Van Waerbeke L., Mellier Y., Schneider P., Jain B., Bernardeau F., Erben T., 2001. A\& A, 368, 766.

Schneider P., Van Waerbeke L., Jain B., Kruse G., 1998. MNRAS, 296, 873.

Schneider P., Van Waerbeke L., Mellier Y., 2002. A\& A, 389, 741.

Smith R., Peacock J., Jenkins A., White S., Frenk C., Pearce F., Thomas P., Efstathiou G., Couchman. H., 2003. MNRAS, $341,1311$.

Spergel D., Verde L., Peiris H., WMAP Collaboration, 2003. ApJ, submitted, astroph/0302209,

Taylor A., 2001. Phys. Rev. Lett, submitted, astroph/0111605, .

Tegmark M., Zaldarriaga M., 2002. Phys. Rev. D, 66, 103508.

van den Bosch F. C., Abel T., Croft R. A. C., Hernquist L., White S. D. M., 2002. ApJ, 576, 21.

Van Waerbeke L., Mellier Y., Radovich M., Bertin E., Dantel-Fort M., McCracken H., Fevre O. L., Foucaud S., Cuillandre J., Erben T., Jain B., Schneider P., Bernardeau F., Fort B., 2001. A\& A, 374, 757.

Van Waerbeke L., Mellier Y., Pello R., Pen U.-L., McCracken H., Jain B., 2002. A\& A, 393, 369.

Wilson G., 2003. ApJ, 585, 191.

Wolf C., Dye S., Kleinheinrich M., Rix H., Meisenheimer K., Wisotzki L., 2001. A\& A, 377, 442.

\section{APPENDIX A: OPTIMAL GALAXY PAIR WEIGHTING}

For a pair of galaxies with estimated redshifts $\hat{z}_{a}$ and $\hat{z}_{b}$ and associated errors $\Delta_{z}$, following Heymans \& Heavens (2003), we assign a zero weight if $\left|\hat{z}_{a}-\hat{z}_{b}\right|<\alpha \Delta_{z}$ and a weight of one otherwise. We choose $\alpha$ to minimise the total error on the shear correlation function which has two components, one systematic error from intrinsic galaxy alignments, $\sigma_{\mathrm{IA}}$, and one random error from shot noise. The optimum $\alpha$ value will depend on angular separation $\theta$, the COMBO-17 redshift distribution, $\phi_{z}(z)$, shown in Fig. A1, and the median photometric redshift accuracy, $\Delta_{z}=0.042$. The shot noise comes from the COMBO-17 intrinsic distribution of galaxy ellipticities, $\sigma_{e}=0.67$. The estimated value for $\sigma_{\text {IA }}$ depends on which $\eta(r)$ model we choose. The conservative approach is to consider the highest amplitude intrinsic alignment model, thereby ensuring all possible pairs of galaxies that could contribute to the contaminating intrinsic alignment signal are removed. It is this approach that we use in Section 4.1 in order to observationally constrain the intrinsic alignment contribution to COMBO-17. By doing this however there is the expense of a potentially needless high residual shot noise, and therefore the preferred approach which we use in Section 4.3 , is to use the upper limit of $\eta_{\mathrm{C} 17}(r)$ in order to optimally remove the intrinsic alignment contamination from the weak lensing signal in COMBO-17. 


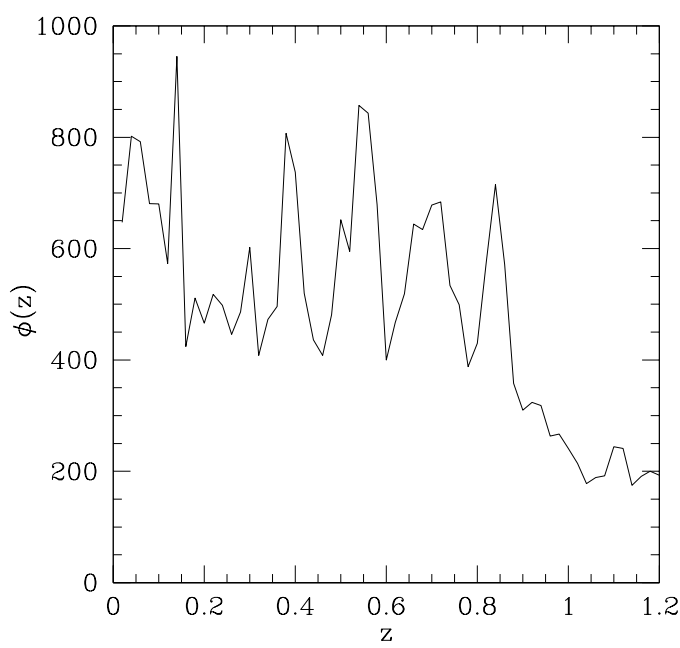

Figure A1. COMBO-17 total redshift distribution for the A901, CDFS and S11 fields, limited to $R<24$, with known galaxy cluster members removed.

Fig. A2 shows the optimal $\alpha$ values calculated for the COMBO-17 redshift distribution for the highest amplitude intrinsic alignment model: Jing (circles), and the upper limit COMBO17 model (stars). Spikes in the COMBO-17 redshift distribution, shown in Fig. A1, mean that $\alpha(\theta)$ is not a smooth function. The higher amplitude Jing intrinsic alignment model requires more galaxy pairs to be rejected than for the lower amplitude COMBO17 model, where the intrinsic alignment contribution is of similar amplitude to the shot noise. In this case the best results are obtained with the rejection of only the closest galaxy pairs but this would not necessarily be the case for large area surveys where high galaxy number counts reduce the total shot noise.

We derive $\alpha$ values for the Jing model considering no evolution in galaxy clustering (solid) for Section 4.2 and considering stable clustering (dashed) for Section 5.2. The angular correlation signal from intrinsic galaxy alignments are less when we include galaxy clustering evolution, therefore the optimal $\alpha$ values are less. The expected intrinsic alignment ellipticity correlation signal with stable clustering, is fairly constant for angular scales $\theta<10$ ar$\mathrm{cmin}$ and this is reflected in the optimal $\alpha$ values. As the shot noise decreases with increasing $\theta$, the optimal $\alpha$ can increase to remove more of the intrinsic alignment signal without increasing the total error.

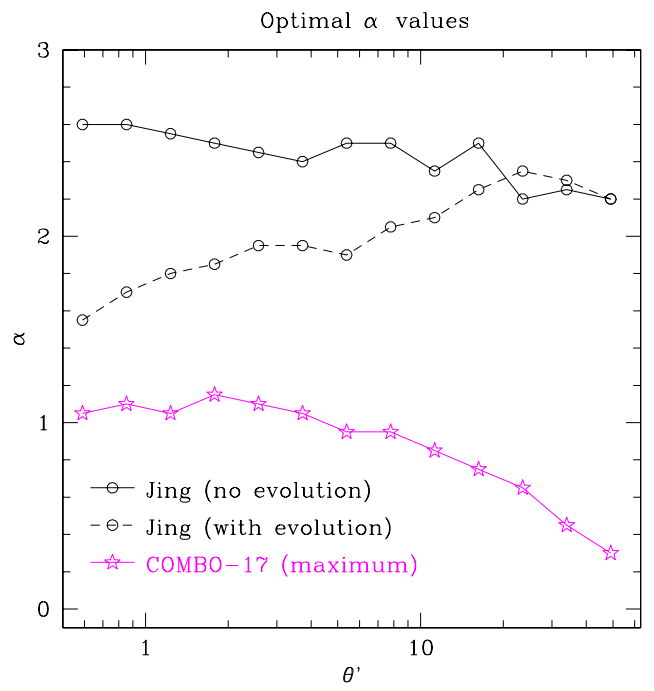

Figure A2. Optimal $\alpha$ values for the COMBO-17 redshift distribution for the Jing intrinsic alignment model (circles), and the upper limit from the COMBO-17 constrained model (stars). For the Jing model we show the optimal $\alpha$ values considering two different galaxy clustering models: zero evolution (solid) and stable clustering (dashed) 